The processes of farming settling of Acámbaro-

Maravatío's region during the i6th century

KARINE LEFEBVRE. Es doctora en arqueología por la Universidad Paris I. Actualmente es Investigadora Asociada en el Centro de Investigación en Geografía Ambiental de la UNAM, campus Morelia. Sus líneas de investigación actuales son la reconstitución de las formas del paisaje, el cambio de uso de suelo en una perspectiva histórica y las relaciones sociedad - medio ambiente. Entre sus últimas publicaciones destaca: en prensa "De lo efímero a lo perdurable, el sello de la religión cristina en el paisaje: el sistema constructivo de los edificios religiosos primitivos", Relaciones.

RESUMEN. Rápidamente después de la conquista de los antiguos reinos prehispánicos, empezó la fase de colonización de estos "nuevos" territorios. Para fomentar el establecimiento de los colonos, la Corona repartió grandes superficies de tierra para vocación agrícola o ganadera. Sin embargo, a pesar de este apoyo, a una escala local, la difusión de la población española y la colonización agropecuaria hispánica siguieron temporalidades y procesos distintos. Varios criterios influyeron sobre el avance: las características geográficas de los sectores, el patrón de asentamiento prehispánico, y también las características propias de cada terruño y los acondicionamientos preexistentes que podían explotar y apropiarse los colonos.

PALABRAS CLAVE. Colonización agraria, patrón de asentamiento, economía, apropiación territorial, transformación del paisaje, Michoacán

ABSTRACT. Soon after the conquest of the pre-Hispanic kingdoms, the phase of colonization in the "new" territories begun. The Spanish Crown distributed large areas of land for agricultural or livestock production to promote the establishment of the colonists. In spite of there being support, the spread of both the Spanish population and the Hispanic agricultural colonization followed different processes, at the local scale. Several criteria influenced the advancement of land occupation such as geographical features of the sectors, the patterns of pre-Hispanic settlements, as well as the features of each native landscape, and the preceding land conditions that could be exploited and appropriated by the settlers.

KEYWORDS. Agrarian colonization, settlement pattern, economy, territorial appropriation, landscape transformations, Michoacán

INFORMACIÓN DEL ARTÍCULO

Recibido: 18 de abril de 2017

Aprobado: 21 de junio de 2018

Correo para correspondencia: karine.lefebvre07@gmail.com 


\title{
Los procesos de colonización agropecuaria de la región de Acámbaro-Maravatío durante el siglo XVI ${ }^{\mathrm{I}}$
}

\author{
KARINE LEFEBVRE
}

Consumada la conquista militar de las provincias mesoamericanas, empezó otra fase: la colonización de los “nuevos” territorios. Esta etapa se caracterizó por la llegada "masiva" y el establecimiento de poblaciones hispánicas: en un primer momento, se instalaron los conquistadores, que rápidamente reunieron a sus familias que se habían quedado en España o en el Caribe, y poco tiempo después, el fenómeno se acentuó con la venida de españoles particulares. Los recién llegados estaban decididos en sacar provecho de los nuevos territorios. La esperanza de riqueza y la búsqueda de un mejor estatus social no eran características propias de los conquistadores, todos los colonos que vinieron y se instalaron en estos territorios tenían la expectativa de una vida mejor, en particular a través de la explotación minera y de la colonización agraria. Por otra parte, la Corona española necesitaba la implantación de sujetos procedentes de España para ocupar sus nuevas provincias y por este conducto, facilitar la instauración de su poder y de su autoridad sobre los territorios y sus poblaciones. Desde luego, los colonos se volvían una "herramienta" del rey en el proceso de colonización.

Después de la Conquista, el sistema indígena de tenencia de la tierra sufrió profundas transformaciones. Como lo menciona T. Jalpa Flores (2008: 112), "Muchas de las [tierras] de carácter público [las de uso común, las destinadas

1 Este artículo es una versión revisada del capítulo III.2.1 de la tesis de doctorado de la autora (Lefebvre, 2012), completada por informaciones obtenidas en el marco del proyecto "Entre campos y corrales, génesis del terruño colonial de Acámbaro, siglos XVIXVII", realizado durante una estancia posdoctoral en el Centro de Investigaciones en Geografía Ambiental de la UNAM entre 2014 y 2016. 
al funcionamiento de los templos, las de los “funcionarios públicos” y las del Estado] se declararon realengas y otras se incorporaron como patrimonio de los cacigazgos". Las autoridades hispánicas reconocieron cuatro tipos de tierras en los territorios conquistados: en primer lugar, las tierras que pertenecían a la nobleza indígena la cual conservaba sus derechos hereditarios, después, las tierras de las comunidades indígenas, luego, el patrimonio familiar y por último, las tierras realengas. Desde la etapa inicial de la colonización, la Corona transfirió a las autoridades novohispanas el poder de conceder las tierras en su posesión. En un principio, Hernán Cortés distribuyó las encomiendas, así como tierras a sus conquistadores. Después, esta prerrogativa fue transferida a la Real Audiencia, antes de ser delegada al Virrey (Jalpa Flores, 2008: 125-126). Así, al inicio, las concesiones jugaron un papel de compensación para los conquistadores por los servicios prestados a la Corona durante la Conquista, y posteriormente, éstas se convirtieron en un instrumento para favorecer el establecimiento de los colonos. Las concesiones realengas fueron de diferentes tipos: sitios de estancia para ganado menor (cabras, ovejas, puercos) o mayor (vacas y caballos), caballerías de tierra destinadas a la agricultura, concesión de agua, además de sitios para establecer ventas, ingenios, molinos². Según la ley, cualquier persona podía solicitar una merced, con la condición de que no existiera ninguna molestia para los propietarios vecinos, sobre todo para las comunidades indígenas cuyas tierras estaban protegidas. Además, los solicitantes debían cumplir con algunas obligaciones, por ejemplo, la de poner el terreno otorgado en explotación, de no venderlo antes de que pasaran seis años y de no venderlo a ninguna iglesia o persona eclesiástica.

La llegada "masiva" de colonos españoles y la repartición de las tierras tuvieron impactos mayores, tanto sobre la organización social de las poblaciones, como en la economía regional y en los paisajes, los cuales mutaron rápidamente, en apenas algunas décadas. En efecto, los colonos traían con ellos

2 Una función específica era asignada a cada tierra otorgada y este uso debía de ser respetado. De esta función dependía el tamaño de la parcela concedida: una superficie de 43 ha para las caballerías, 780 ha para las estancias para ganado menor y 1755 ha para las de ganado mayor. Si el propietario quería cambiar su dedicación tenía que hacer una solicitud especial ante la Real Audiencia. 
nuevas especies vegetales y animales, técnicas y esquemas de organización agropecuaria, heredados de las tradiciones europeas y mediterráneas, distintos de los aplicados por las poblaciones indígenas. Además, las nuevas orientaciones económicas influyeron sobre otros sectores: tales como las dinámicas de poblamiento, el lugar y el papel de las distintas clases de la sociedad dentro del sistema productivo.

Desde hace varias décadas, se abrió un gran debate, entre investigadores de historia, de geografía y de historia ambiental, enfocado sobre la importante alteración en el paisaje ocasionada por la colonización española. Las investigaciones se enfocaron en distintos aspectos del cambio de paisaje y condujeron a conclusiones diferentes sobre el origen de la degradación del ambiente: el desarrollo de las actividades agrícolas de los siglos XVIII y XIX (Butzer y Butzer, 1993), la cría intensiva, y en particular la gran densidad de ovejas durante el siglo XVI (Melville, 1994), una importante sequía (Endfield y O’Hara 1999), a la cual Hunter (2009) asocia el abandono de las terrazas indígenas, por mencionar sólo algunos. En cambio, fueron menos numerosas las indagaciones que se enfocaron sobre la influencia del paisaje - a la vez sus componentes naturales y antrópicas - para entender el esquema de difusión de la colonización agropecuaria hispánica del primer siglo de la colonización. Las interacciones sociedades-paisajes son simultaneas, y si bien las sociedades afectan y transforman el paisaje en el cual evolucionan, en particular a través de sus actividades socioeconómicas, también están afectadas por él (Worster, 1990). Como lo explica C. Sauer (1925: 6), el paisaje "Podría ser definido, por tanto, como un área compuesta por una asociación distintiva de formas, tanto físicas como culturales”. Más allá de sus características físicas, cada paisaje fue modelado, transformado a lo largo de los siglos por las distintas sociedades que en él se sucedieron. En función de su organización y de sus necesidades socio económicas, pero también de sus relaciones al medio ambiente, estas últimas marcaron los paisajes con la construcción de acondicionamientos, aprovecharon instalaciones preexistentes, o cuando fuera necesario las transformaban o las destruyan. Desde entonces, se llevaron a cabo procesos de continuidad de algunos elementos y formas estables en el tiempo, el abandono de algunos 
componentes y la aparición de nuevos, participando así en la formación de terruños singulares y de paisajes culturales.

Desde entonces, el peso de las estructuras sociales y económicas prehispánicas preexistentes (patrón de asentamiento, patrón y acondicionamientos agrícolas, vías de comunicación ...) junto a las características naturales del entorno (relieves, geología, cuerpos de agua, vegetación...) influenciaron la propagación de los colonos españoles (ejes de difusión, intensidad y temporalidades) y la formación de los paisajes agropecuarios coloniales.

La compleja situación del territorio de Acámbaro, ubicada en los límites de los estados actuales de Guanajuato y de Michoacán y al margen del área mesoamericana (al momento de la Conquista), constituye un marco de estudio particularmente interesante para entender las políticas de colonización agraria establecidas por el poder central. En víspera de la Conquista, el territorio de Acámbaro-Maravatío era una frontera del reino tarasco frente al imperio mexica al este y a los territorios chichimecas al norte. El patrón de asentamiento y la política de gestión de las poblaciones locales fueron estructurados por el poder central tarasco con el fin de proteger la región de las incursiones mexicas, y por ende una vía de acceso natural hacia el corazón del reino. La ocupación tarasca se concentró en la orilla izquierda del río Lerma, mientras que la orilla derecha quedó "vacía de población", sirviendo de "barrera" de separación entre las dos potencias político-militares (Lefebvre, 2012). La diferencia de contexto entre las orillas izquierda y derecha del río Lerma tuvo consecuencia en el desarrollo de la colonización ya sea en sus aspectos cronológicos como en la forma en que se dio. La existencia de un paisaje antropizado en la orilla izquierda, marcado por la presencia de pueblos, de una amplia red de caminos, de terrazas agrícolas en las faldas de los cerros, de parcelas trabajadas y de canales de irrigación, es decir de un terruño activo con estructuras agrícolas en funcionamiento, y por lo contrario, la deserción de la orilla derecha desde por lo menos un siglo y medio, y por lo tanto el abandono y la aniquilación progresiva de los acondicionamientos agrícolas de las sociedades que allí habían vivido, ofrecían dos contextos totalmente distintos para el reparto y la explotación de las tierras. Por otro lado, la región de Acámbaro-Maravatío, verdadero mosaico geográfico 
compuesto de sierras altas y de cerritos aislados entre los cuales se extienden amplios valles resultado de la depresión de los ríos, ofrecía paisajes más o menos favorables al establecimiento de una actividad agropecuaria extensiva.

A partir del ejemplo concreto de la colonización agropecuaria de esta región, se analizará el proceso de reparto de las tierras, tomando en cuenta la evolución a la vez temporal y espacial de la política de distribución. Se buscará entender: ¿Cuál fue el esquema de difusión de las poblaciones hispánicas?, ¿Cuáles son los factores que favorecieron o, a contrario, frenaron la colonización agropecuaria?, ¿Frente a la apropiación de las tierras por los colonos, cuál fue la respuesta indígena? De esta manera, se podrá medir la influencia de la situación prehispánica y de las características geográficas sobre los procesos de colonización agraria y el cambio de tenencia de la tierra.

DE LA DOCUMENTACIÓN HISTÓRICA

El desarrollo económico fue uno de los principales motores de la colonización, por lo tanto las fuentes históricas de los siglos XVI y XVII proporcionan una base documental de primer orden para el estudio del desarrollo económico y de las transformaciones de los paisajes del México colonial. La mayoría de las informaciones exploradas en la presente investigación proceden de la documentación administrativa, y en particular de los ramos Mercedes y Tierras del Archivo General de la Nación (AGN, México).

Durante el siglo XVI, las donaciones de tierras realengas a los colonos europeos, a los miembros de las antiguas noblezas indígenas, e incluso a las comunidades, fueron registradas por la administración central según un estricto protocolo. La mayoría de los actos que confirman estas posesiones está conservada en el ramo Mercedes. Estos documentos se conforman de una primera parte que indica el destinario, la función de la tierra y una descripción más o menos sintética de la ubicación de la(s) parcela(s) y enseguida, una parte legal muy estereotipada que menciona las obligaciones del nuevo posesor.

Otras informaciones referente a la repartición de las parcelas están guardadas en el ramo Tierras. Este ramo contiene documentos, menos estandarizados, que incluyen las solicitudes y las investigaciones previas a la donación de 
las tierras, mientras que otros relatan litigios territoriales que ocurrieron durante la época colonial ${ }^{3}$.

En total, 228 documentos, fechados de 1542 a 1607 y que corresponden a la región de Acámbaro, Jerécuaro, Zinapécuaro y Maravatío fueron objeto de análisis. Estos documentos se refieren principalmente a la distribución de tierras agropecuarias, y en una menor proporción a la edificación de molinos, derechos sobre el agua, obras de construcción o de reparación de caminos reales o secundarios, que si bien no aluden a tierras productivas, si proporcionan informaciones esenciales para entender la colonización agropecuaria.

Por lo general, los actos (que proceden de los ramos Mercedes o Tierras) estaban acompañados de documentos cartográficos (croquis o pinturas). Estos mapas, que tenían por objetivo localizar los principales elementos del asunto, generalmente eran realizados a una escala muy local e integraban rasgos iconográficos europeos, indígenas o mixtos. Sin embargo, muchos de estos documentos desaparecieron a lo largo del tiempo, y sólo se pudieron registrar 25 en la región de Acámbaro. El valor informativo de estos mapas fue infravalorado durante mucho tiempo, siendo relegados al papel de ilustraciones, pero éstos aportan numerosas informaciones de primer orden acerca de los cambios del paisaje y del uso de suelo. Estos documentos no permiten ubicar cronológicamente la otorgación de todas las tierras, pero proporcionan una imagen (muchas veces parcial) del paisaje en un momento dado, ya que suelen figurar los principales elementos geográficos (cerros, barrancas, ríos...), pueblos coloniales, estructuras agropecuarias, caminos y a veces elementos del patrón de asentamiento y de la cosmovisión prehispánica. Además, ponen de manifiesto la densidad de ocupación y elementos que influyeron en la ocupación agropecuaria.

El análisis del corpus de los documentos conservados en el ramo Mercedes del AGN permite poner de manifiesto la evolución de la repartición espacial y temporal de las tierras otorgadas en el transcurso del siglo XVI. Las informaciones fueron complementadas por los relatos, muchas veces más generales, de

3 Los documentos del ramo Tierras que evocan litigios pueden ser más tardíos (hasta el siglo XVIII), pero se refieren y a veces reproducen actos de donaciones de los siglos XVI-XVII para resolver los conflictos. 
los religiosos y viajeros que cruzaron la región durante el siglo XVI, y por los asuntos comentados en el ramo Tierras.

EVOLUCIÓN TEMPORAL DE LA REPARTICIÓN DE LAS TIERRAS EN LA REGIÓN

El análisis de la repartición de las tierras en el transcurso del siglo XVI permite identificar cinco fases en el proceso de colonización agraria (figura 1).

La primera tierra (por la cual se encuentra un acto de donación oficial) fue otorgada en 1542 a Hernán Pérez de Bocanegra para la edificación de un molino y la implantación de un área de batán en el territorio de Zinapécuaro. Aunque el documento no concede una tierra productiva para vocación agropecuaria, esta primera merced es muy simbólica, porque se refiere a un ilustre personaje: el segundo encomendero de Acámbaro, que posteriormente logró conseguir numerosos otros terrenos, hasta formar un verdadero imperio agropecuario.

Es posible, e incluso seguro, que otras tierras hayan sido otorgadas antes de 1542, sin que se encontraran constancias de su repartición. En efecto, se hallan documentos de la década de 1540 o principios de los años 1550, que mencionan - para fijar los límites de las tierras otorgadas - la existencia de propiedades en manos de colonos españoles ya instalados en la región, de las cuales no existe mención de su adquisición. Por ejemplo, en 1554, Beatriz Hernando recibe una parcela destinada a la cría de ganado mayor en Maravatío ${ }^{4}$. El terreno es contiguo a las estancias de Alonso Ávila y del licenciado Altamyrano. Aunque la tierra de Beatriz Hernando fue otorgada 12 años después de la de Bocanegra, se trata de la segunda merced registrada en la región, y no se encontró referencia de cuándo, por quién y por qué medio se concedieron las tierras a Ávila y a Altamyrano.

De la misma manera, dos documentos tempranos evocan litigios existentes entre comunidades indígenas y propietarios españoles. El primero, de 1555, hace referencia a un conflicto enfrentando indios de Maravatío y españoles 5 .

4 AGN, Mercedes, vol. 4, exp. 198, f. 62.

5 AGN, Mercedes, vol.3, f. $199 \mathrm{v}$. 
El segundo, fechado del mismo año, evoca los daños ocasionados por el ganado mayor en los campos de la comunidad de Qunoneo, pueblo sujeto de Acámbaro $^{6}$. Si bien el documento no menciona de manera directa a terratenientes españoles, cabe recordar que la cría de ganado mayor era una prerrogativa de los colonos europeos. Así, estos testimonios hacen claramente alusión a su establecimiento temprano en estos territorios y a la existencia de una actividad agropecuaria hispánica desde los años 1550, sin que se encuentren los documentos de donación.

Por consiguiente, durante la primera fase de la "invasión" europea identificada, que se extiende de 1542 hasta 1559 (figura 1), la repartición de tierras parece ser bastante débil. Apenas se registraron 11 mercedes, de las cuales sólo tres se refieren directamente a tierras productivas. Sin embargo, es importante tomar este dato con cautela, ya que algunas de las fuentes se han podido perder (o nunca fueron redactadas): en razón de la ausencia de numerosos documentos, es imposible cuantificarla con certeza. Esta situación no es propia a la región de Acámbaro, T. Jalpa Flores (2008:132) establece una constatación similar en la de Chalco (Estado de México). En efecto, durante las primeras décadas que siguieron la Conquista, el proceso de repartición de las tierras no fue objeto de un registro oficial riguroso, e incluso parece soltarse, por lo menos en parte, del poder central, lo que explica el silencio de las fuentes durante las dos primeras décadas de la colonización.

Una segunda fase, de 1560 a 1568, está marcada por un primer auge del reparto de las tierras, ya que en solamente ocho años, 32 mercedes fueron otorgadas (figura 1), dentro de los cuales 28 fueron para colonos españoles. De nuevo el número real de concesiones debe de ser subestimado, puesto que las descripciones siguen mencionando a vecinos españoles, de los cuales se desconocen las condiciones de adquisición de sus tierras. Esta etapa, que anuncia el principio de la colonización agropecuaria europea, ocurre tras la primera gran fase de congregación de las poblaciones indígenas (1550 - 1564). En efecto, el siglo XVI está marcado por dos procesos distintos de reorganización del patrón de asentamiento prehispánico: el desplazamiento de los

6 AGN, Mercedes, vol. 4, f. 282. 
asentamientos prehispánicos y las congregaciones de los pueblos. Recientes estudios arqueológicos llevados a cabo en la región de Acámbaro-Maravatío demostraron que en víspera de la Conquista española, el patrón de asentamiento se caracterizaba por pequeños pueblos dispersos, generalmente establecidos en las alturas (cima y pendientes altos de los cerros), en posición estratégica para defender las fronteras del reino tarasco contra las incursiones mexicas y chichimecas (Lefebvre, 2012). Frente a esta situación, los españoles trasladaron los sitios desde las alturas hacia los valles o planicies cercanos (generalmente a una distancia inferior a un $\mathrm{km}$ ), lo anterior con el fin de evitar que los antiguos asentamientos se convirtieran en bastiones y en focos de rebelión (Musset, 2002). En segundo lugar, a lo largo del siglo XVI, los españoles realizaron fases de reagrupamiento de las poblaciones, denominadas "Congregaciones" (la primera entre 1550 y 1564, y la segunda entre 1593 y 1605). Esta política de gestión de las poblaciones, instaurada por el poder central, consistía en agrupar a los habitantes de los pueblos pequeños y lejanos o de los que habían sido despoblados a causa de las epidemias o del trabajo forzoso (servicio personal) en asentamientos mayores para facilitar el control de las poblaciones, su evangelización y la recaudación del tributo (Fernández-Christlieb y Urquijo, 2006; Reyes García, 2003; Torre Villar, 1995; entre muchos otros). Según la ley española, las comunidades indígenas conservaban los derechos sobre sus tierras ancestrales. En la región de Acámbaro - Maravatío, entre 1560 y 1563, cuatro concesiones fueron otorgadas a comunidades indígenas y una a un cacique; probablemente resultan de un proceso de regularización de tierras comunales (y no del otorgamiento de nuevos terrenos), concediendo propiedades a los pueblos desplazados o congregados, o confirmando antiguas posesiones. Sin embargo, en los hechos, para muchos pueblos, esta restructuración del espacio colonial se acompañó del abandono de sectores agrícolas enteros, muchas veces por estar demasiado alejados de los nuevos asentamientos. Por otra parte, las grandes epidemias que se sucedieron desde la llegada de los españoles ${ }^{7}$ diez- $^{-}$ maron pueblos enteros, dejando sus tierras agrícolas "libres" (Jalpa Flores,

7 Por mencionar sólo algunas : la viruela en 1519-1520, el sarampión y la varicela en 1531, la rubeola en 1538-1539, el tifus, la tifoidea y la peste a partir de 1545 . 
2008: 133 ; Cook y Simpson, 1948). Desde entonces, los colonos aprovecharon esta situación para ocupar terrenos fértiles y, algunas veces, equipados de red de irrigación. Numerosos ilustres personajes de la Conquista y sus familiares cuentan entre los españoles que obtuvieron tierras durante este periodo. En particular, es el caso de Hernando Pantoja (hijo del conquistador Pedro Pantoja y cuñado de Gaspar Morcillo, encomendero de Indaparapeo) ${ }^{8}$, de Luis Ponce de León y Bernardino Pacheco, hijos de Hernán Pérez de Bocanegra (segundo encomendero de Acámbaro) ${ }^{9}$, de Lope Cendejas (hijo del conquistador Pedro Moreno $)^{10}$. También miembros de la administración local aprovecharon de la redistribución de las tierras indígenas, tal como es el caso de García Monroy ${ }^{11}$, justicia de Acámbaro, por mencionar sólo uno. Los familiares de los conquistadores además de obtener cargas administrativas como las de encomenderos, jueces, alcaldes mayores o corregidores, logran poner la mano en amplias extensiones de tierras, volviéndose así los primeros terratenientes.

Más adelante, entre 1569 y 1580 (tercera fase), las donaciones de tierra disminuyeron de manera brusca e importante, ya que sólo se encontraron dos documentos de donación para este periodo (figura 1). Este declive de la colonización agropecuaria española fue de corta duración. La cuarta fase, desde 1581 hasta 1596, está marcada por un nuevo auge de la repartición de las tierras por el Virrey, sin parangón con la precedente, ya que se registraron 147 actos de mercedes, correspondientes a 276 caballerías de tierra, 34 estancias de ganado mayor, 110 de ganado menor, un ingenio de azúcar, siete ventas y cinco molinos. En 15 años, más de 157,000 hectáreas de tierra fueron distribuidas: una superficie gigantesca. T. Jalpa Flores (2008: 134), quien observa una evolución similar en la región de Chalco entre 1594 y 1616, atribuye este aumento considerable a la propagación epidémica y a la segunda fase de congregación. En la región de Acámbaro-Maravatío, esta fase de otorgación masiva es más precoz y por lo tanto no puede ser atribuida a la política de

8 AGN, Mercedes, vol. 7, f. 104, AGN, Mercedes, vol. 7, f. 128.

9 AGN, Mercedes, vol. 7, fs. 120-120v ; AGN, Mercedes, vol. 9, fs. 132-132v.

10 AGN, Mercedes, vol. 7, f. 264.

11 AGN, Mercedes, vol. 8, f. 82. 


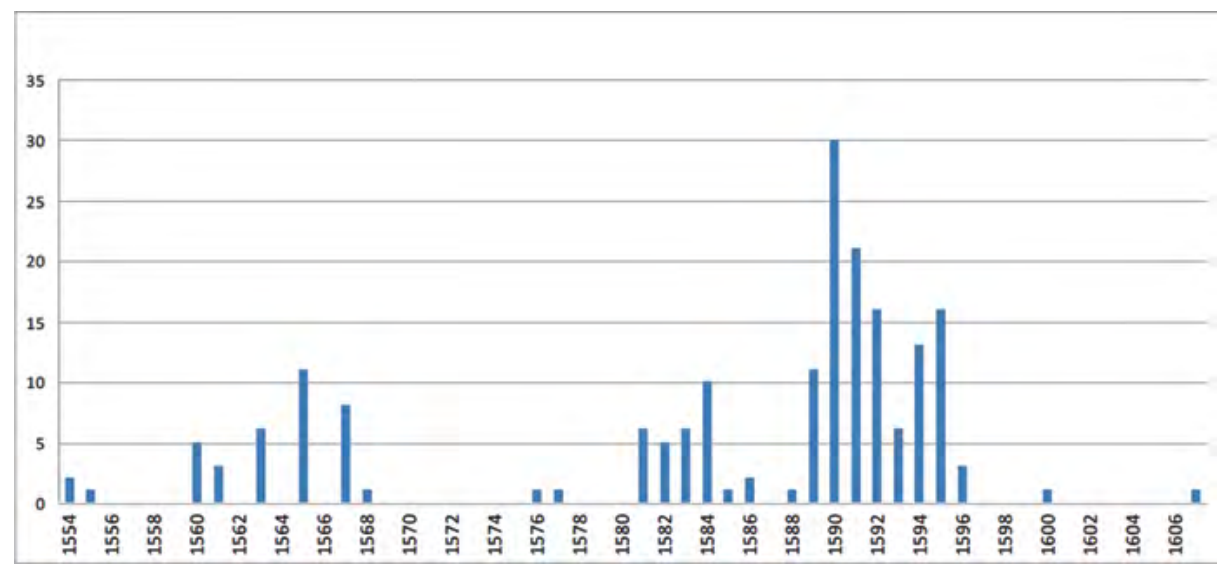

Figura I. Evolución cronológica de la distribución de las mercedes (número de donación por año)

reorganización del patrón de asentamiento impuesta por los españoles. En cambio, en esta época, las epidemias ya habían tenido efectos importantes en la organización espacial de los asentamientos. La Relación Geográfica de Acámbaro $^{12}$, escrita en 1580 , subraya que "Es provincia de dos mil y seiscientos vecinos, [y] ha sido, antes de ahora, muy más poblada. Y por causa de una pestilencia que hubo general en esta Nueva España habrá cuatro a[ñ]os se disminuyó y bajo [la población] en esta d[ic]ha cantidad" (Acuña, 1987: 60). La Relación Geográfica de Taymeo, fechada de 1579, refuerza esta constatación señalando que "Solía ser este pueblo, antiguamente, de más cantidad de gente, y, con la pestilencia pasada al estado en que al presente está” (Acuña, 1987: 277). Pero cabe subrayar que los enfrentamientos con algunos españoles también serían una causa de esta caída demográfica. En 1591, los habitantes del pueblo de San Bartolomé, sujeto de Indaparapeo, se quejaron de los daños del ganado español en sus campos e indicaron que "por no lo poder sufrir se ba disminuyendo el

12 Las Relaciones Geográficas son el resultado de un encuesta realizada en 1577, a petición de Felipe II, con el fin de establecer un inventario preciso de los recursos naturales y humanos, de los asentamientos indígenas y españoles, civiles, militares y religiosos, de las vías de comunicación en sus "nuevos territorios". 
$\mathrm{d}[\mathrm{ic}]$ ho pu[ebl]o" ${ }^{13}$. Los efectos de las epidemias y de los conflictos fueron tales que varios documentos históricos hacen referencia a los asentamientos despoblados y abandonados. Por ejemplo en 1592, se otorga a Agustín Ynojosa un sitio de estancia "junto a un pueblo despoblado nombrado San Lucas que era sujeto del d[ic]ho pu[ebl]o de Acámbaro"14. De igual manera, el 12 de mayo de 1595, se concede a Pedro Gonzales dos tierras ganaderas en el territorio de Acámbaro, en donde se encuentran "[...] unas casillas caydas que a mucho tiempo questan despobladas que eso solían llamar Santa Catalina" ${ }^{15}$. Siguiendo el mismo esquema, en 1599, Diego López Cavallero obtiene tierras en el territorio de Maravatío en “[...] un sujeto suyo llamado Paquizuato [Paquisihuato] questa despoblado" ${ }^{16}$. Si bien no tenemos informaciones acerca de los poblados de Santa Catalina ${ }^{17}$ y Paquisihuato, la Relación Geográfica de Acámbaro (Acuña, 1987: 62) menciona a San Lucas, lo que proporciona un terminus postquem en 1580 (fecha de la redacción del documento). Así, su abandono sucede entre 1580 y 1592. En el caso de Santa Catalina (sujeto de Acámbaro), que no aparece en esta relación, puede haber desaparecido antes de esta fecha. Un documento cartográfico, fechado de 1578, proporciona informaciones similares para el territorio de Zinapécuaro, con la representación de una casa y de unas estancias abandonadas ${ }^{18}$. Por lo tanto, aparece que la repartición masiva de las tierras es la consecuencia directa del abandono de los asentamientos, lo que explica que una amplia mayoría de estas tierras fueron adquiridas por españoles (117 de 147), mientras que solo 17 fueron concedidas (o confirmadas) a principales y 13 a comunidades. Durante esta fase, los encomenderos, los conquistadores, los que tenían una carga administrativa importante, y su familia, siguieron obteniendo numerosas mercedes reales. María Vargas (viuda del

13 AGN, Indios, vol. 5, exp. 962, fs. 317v-318.

14 AGN, Mercedes, vol. 19, fs. 83-83v.

15 AGN, Mercedes, vol. 20, fs. 71v-72.

16 AGN, Mercedes, vol. 19, exp. 456, fs. 163v-164v.

17 La única información viene del hagiotoponimo del poblado que demuestra una cristianización del lugar y por lo tanto una ocupación a principio de la época colonial.

18 AGN, Tierras, vol. 2737, exp. 11, f 25, sección mapas 1956. 
encomendero de Indaparapeo Gaspar Morcillo $)^{19}$, Hernando Pacheco ${ }^{20}$, Nuño de Chávez Bocanegra ${ }^{21}$ y Francisco Pacheco de Bocanegra ${ }^{22}$ (tres hijos del encomendero Hernán Pérez de Bocanegra), o también Nicolás de Castro, interprete de la Real Audiencia ${ }^{23}$, recibieron tierras, a veces en gran cantidad. El ejemplo más característico es el de Nuño Chávez de Bocanegra, heredero de la encomienda de su padre, que obtuvo cinco estancias para ganado mayor y 10 caballerías de tierra, lo que representa 9,205 ha. Pero, en paralelo de estos propietarios de alto rango social, aparece y se amplifica una nueva clase de terrateniente, compuesta de particulares, de colonos que no participaron en la Conquista o en el establecimiento de la administración novohispana, atraídos por la esperanza de una vida mejor y de riqueza. No se identificaron vínculos entre estos individuos y la alta administración o los conquistadores de primera hora. Así, esta cuarta fase parece marcada por la llegada "masiva" de colonos españoles, que no participaron en la Conquista.

Finalmente, a partir de 1596, se observa un declive brusco de las donaciones de tierra, hasta 1607. Durante estos 11 años, solo cuatro mercedes fueron identificadas.

\section{LA REPARTICIÓN DE LAS TIERRAS POR CABECERA}

Durante el siglo XVI, la región de Acámbaro se divide en seis unidades territoriales administrativas encabezadas por un asentamiento mayor: Acámbaro, Aráro-Zinapécuaro, Taimeo, Ucareo, Maravatío e Indaparapeo (figura 2). La repartición de las tierras otorgadas en cada cabecera atestigua de importantes disparidades (figura 3). Algunos sectores fueron particularmente afectados, mientras que otros parecen quedarse fuera del proceso de colonización agraria.

19 AGN, Mercedes, vol. 18, exp. 131, f. 42v ; AGN, Mercedes, vol. 18, f. 96v.

20 AGN, Mercedes, vol. 17, exp. 799, fs. 232-232v.

21 AGN, Mercedes, vol. 18, exp. 463, f. 116.

22 AGN, Mercedes, vol. 18, fs. 154v-155.

23 AGN, Mercedes vol. 16, exp. 572, fs. 153-153v ; AGN, Mercedes, vol. 16, exp. 321, fs. $89 \mathrm{v}-90$. 


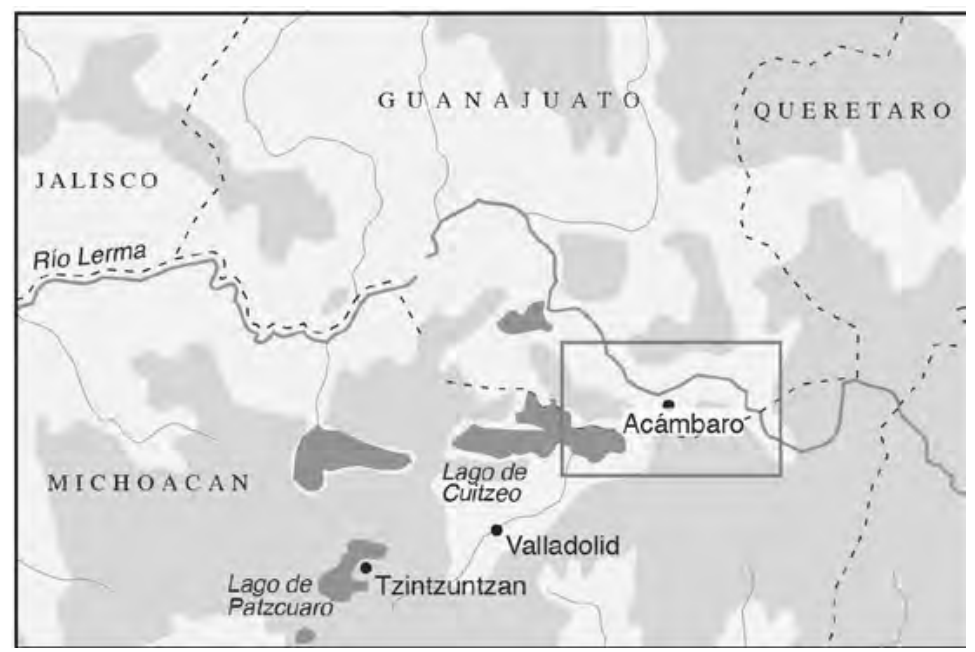

Figura 2. Extensión de los territorios de las cabeceras durante el siglo XVI, según los datos históricos

Por ejemplo, a lo largo del siglo XVI, el sector de Ucareo solamente cuenta con tres donaciones de tierra registradas, entre las cuales sólo un documento deja constancia de la concesión de tierras ganadera a un colono, en 1565. Las dos otras mercedes corresponden a la confirmación de derecho o protección de tierras perteneciendo a comunidades indígenas, lo que es una prueba indirecta de la presencia de españoles en el sector.

Los territorios de Taimeo y de Indaparapeo, aunque fueron un poco más favorecidos, tuvieron un destino cercano. Para ambos sectores, la primera merced registrada fue con fecha de $1560^{24}$. Siguiendo una misma trayectoria, entre 1581 y 1595 , ambas jurisdicciones están marcadas por un aumento de las donaciones. Durante la cuarta fase, se otorgan en los territorios de Taimeo y de Indaparapeo, respectivamente 19 y 16 mercedes. Estas tierras fueron casi exclusivamente entregadas a colonos españoles ( 18 de 19 mercedes en Taimeo y 14 de 16 en Indaparapeo). Las escasas mercedes concedidas a poblaciones

24 AGN, Mercedes, vol. 5, fs. 110-110v, AGN, Mercedes, vol. 5, fs. 121-121v. 
indígenas, o más bien, el reconocimiento de sus terrenos, podrían sugerir de una respuesta débil de las comunidades frente a la invasión española, la cual parecía muy poco marcada. Sin embargo, caben resaltar dos elementos que matizan esta hipótesis. En un mapa fechado en $1578^{25}$ figura una porción de tierra que se extiende desde los contrafuertes de la Sierra de Ucareo hasta la laguna de Cuitzeo y presenta una verdadera dicotomía del espacio (figura 4). Los sectores altos están representados por relieves pintados de color verde y cubiertos por numerosos árboles que, aunque figurados de manera esquemática, se pueden identificar como árboles resinosos. En cambio, en los sectores bajos están establecidas varias unidades agropecuarias, en una zona pantanosa contigua a la laguna de Cuitzeo y cerca del camino real que lleva a la ciudad de Valladolid. Otro documento cartográfico fechado en $1590^{26}$ también representa este sector, y al igual que el precedente, en él figuran varias unidades agropecuarias. Los dos mapas coinciden en el origen español de los terratenientes. Por consiguiente, aunque no aparece en la documentación textual, esta zona de llanura fue objeto de repartición agraria, y los terrenos fueron adquiridos por unos cuantos individuos hispánicos, mientras que el sector montañoso del territorio de Taimeo quedó sin repartirse y con una economía probablemente más enfocada en la explotación de los bosques (tal vez por las poblaciones indígenas). De la misma manera, en el territorio de Indaparapeo, los pleitos constantes de las comunidades en contra de los españoles en general, y de la familia del encomendero en particular, atestiguan de una resistencia frente a la invasión hispánica.

En el territorio de Maravatío, las concesiones son más tempranas. Desde 1554, Ana Rodríguez Tirado ${ }^{27}$ y Beatriz Hernández ${ }^{28}$ reciben, cada una, una estancia. Pero, a pesar de la precocidad de estos primeros repartimientos, las mercedes (por los cuales se encontraron una constancia) quedaron escasas. De hecho, los dos principales picos de repartición (fase dos y cuatro) fueron muy

25 AGN, Tierras, vol. 3343, exp.15, sección mapas, 2379.

26 AGN, Tierras, vol. 2681, exp.17, sección mapas, 1602.

27 AGN, Mercedes, vol. 4, exp. 218, f. 70v.

28 AGN, Mercedes, vol.4, exp. 198, f. 62. 
poco marcados, puesto que solo se concedieron tres mercedes entre $1550 \mathrm{y}$ .1564 y cuatro entre 1581 y 1595 . Sin embargo, otros terratenientes españoles, algunos muy notables, obtuvieron tierras en el sector. En 1541, Antonio de Mendoza, primer virrey de Nueva España, compró tierras en esta región que probablemente conoció durante su camino a Jalisco para someter a los indios del Mixtón (Ruiz Medrano, 1991: 168-169). Este trato significa que las tierras ya eran en mano de un(os) terrateniente(s), pero ¿se trataba de españoles o de indígenas?

Por último, aunque la información sea muy fragmentada, también sabemos que a finales del siglo XVI, algunas tierras ubicadas en la periferia cercana del pueblo colonial de Maravatío pasaron a manos de Francisco Hernández de Ávila ${ }^{29}$. Más adelante, esas propiedades serían el origen de la formación de la hacienda de Casa Blanca, todavía en manos de la familia a finales del siglo XVIII. La adquisición de estas tierras por Francisco Hernández de Ávila no aparece en el ramo de Mercedes del AGN, y por lo tanto la extensión de estas propiedades no puede ser cuantificada. A la excepción de un expediente referente al conflicto que oponía la familia de Francisco Hernández de Ávila y los naturales de Maravatío, no existen referencias a las tierras indígenas y a las relaciones que las comunidades mantenían con los terratenientes europeos.

La situación es distinta en los territorios de Zinapécuaro y de Acámbaro que fueron particularmente sometidos al proceso de repartición. Se registraron 45 mercedes para Zinapécuaro durante el primer siglo de la Colonia. Como ya se mencionó, la primera merced fue otorgada en 1542 a Hernán Pérez de Bocanegra. Después de esto, hay que esperar casi 20 años, al principio de la década de 1560, para que se registren cuatro donaciones (dos a colonos españoles, una a una comunidad, y la última a un cacique), así como dos protecciones aplicadas a tierras indígenas. Pero, el verdadero incremento de la

29 AGN, Tierras, vol. 1162, exp.9. En las primeras décadas del siglo XVI, el pueblo prehispánico de Maravatío, ubicado a proximidad del poblado actual de Santa Rita (en los contrafuertes de la Sierra de Ucareo), fue desplazado y establecido en la llanura del río Lerma, en un paraje llamado Casa Blanca hoy en día. Casi un siglo después, de nuevo fue traslado (donde se encuentra ahora), en consecuencia de un conflicto entre la comunidad y la familia de hacendados Hernández de Ávila. 
población española sucede durante el segundo pico de repartición, así entre 1581 y 1595 , se otorgaron 41 mercedes. Siguiendo el esquema general, estas donaciones de tierra se concedieron principalmente a españoles (28 de las 41). Sin embargo, las donaciones a las comunidades, los hospitales de Indios y los miembros de la antigua élite indígena no fueron menores. Varios indios, caciques y principales, lograron obtener propiedades: Sebastián Tanga ${ }^{30}$, Diego Tanga $^{31}$, Juan García ${ }^{32}$ y Miguel Ynaqua ${ }^{33}$, Juan Bautista Panbagui ${ }^{34}$. Estas numerosas donaciones de parcelas principalmente destinadas a las manadas demuestran una cierta continuidad del dominio y de las prerrogativas de la antigua élite indígena, y una adhesión nueva de los indígenas y sobre todo de su élite, por la crianza (y el consumo) de ganado europeo. Es posible que en algunos casos los caciques hayan adquirido títulos de terrenos para proteger a las comunidades bajo su mando. Sin embargo, esta explicación no debe de ser generalizada. Los cambios de tenencia de la tierra y las alteraciones sociales convirtieron a los caciques en propietarios particulares. Desde entonces, su uso de las instituciones coloniales para obtener tierras servía a un proyecto personal y por lo tanto reflejaba un distanciamiento de las comunidades. H. Roskamp (2016) atestigua de este alejamiento en Zinapécuaro, en donde un importante litigio opuso el cacique don Alonso Huapean a su comunidad en los años 1566-1567.

En el territorio de Acámbaro, el proceso de repartición de la tierra alcanza su máximo nivel. Entre 1550 y 1607, se dieron 95 mercedes, que corresponden a más de 97,000 ha. La primera merced que otorga tierras está registrada en 1560. Sin embargo, solicitudes de investigación y de protección de las tierras indígenas, emitidas antes de esta fecha, atestiguan de la presencia de colonos en este territorio previamente ${ }^{35}$. Entre 1560 y 1567 (segunda fase), 16 mercedes

30 AGN, Mercedes, vol. 12, fs. 125.

31 AGN, Mercedes, vol. 13, fs. 143v-144.

32 AGN, Mercedes, vol. 13, fs. 231-231v.

33 AGN, Mercedes, vol. 13, fs. 234v-235.

34 AGN, Mercedes, vol. 15, fs. 184-184v ; AGN, Mercedes, vol. 16, exp. 1017, f. 280 ; AGN, Mercedes, vol. 20, f. 179.

35 AGN, Mercedes, vol. 4, f. 282. 
fueron listadas, mucho más que en los demás territorios ${ }^{36}$. Estas propiedades fueron en gran medida otorgadas a españoles. Durante este periodo, solo una tierra fue concedida a un miembro de la antigua nobleza indígena: don Cristoval de León, cacique de Acámbaro ${ }^{37}$. Entre 1581 y 1596, segundo pico de donación, las concesiones se multiplicaron de manera exponencial, ya que se otorgaron 81 mercedes, sobrepasando de lejos los otros territorios. Las concesiones a los españoles siguen siendo mayoritarias, pero las comunidades indígenas estuvieron más presentes que anteriormente, recibiendo tierras ganaderas y de culti$v^{38}$. De la misma manera, los caciques y principales indígenas de Acámbaro siguen siendo gratificados con el reconocimiento de sus antiguas posesiones y con nuevas propiedades. El caso más notable es el de don Diego del Águila, potente cacique de Acámbaro que recibe por lo menos tres sitios de estancia para ganado mayor, dos para el ganado menor y siete caballerías de tierra, que representan más de 7,000 ha ${ }^{39}$. Estas numerosas tierras, reconocidas o adquiridas, demuestran la importancia de estos personajes, que logran tener en sus manos muchas más propiedades que numerosos españoles ${ }^{40}$.

La revisión de la evolución de la repartición de las tierras en cada territorio pone de manifiesto unos puntos en común pero sobre todo destinos divergentes.

A excepción de la donación muy precoz (1542) concedida a Hernán Pérez de Bocanegra, las primeras constancias de mercedes aparecen de manera

36 Durante este periodo, se otorgan dos mercedes en el territorio de Zinapécuaro, una en Ucareo, cinco en Maravatío, cuatro en Indaparapeo, y una en Taimeo.

37 AGN, Mercedes, vol. 8, f. 39.

38 AGN, Mercedes, vol. 13, fs. 80-80v ; AGN, Mercedes, vol. 16, exp. 104, f. 33 ; AGN, Mercedes, vol. 16, exp. 328, fs. 92-93 ; AGN, Mercedes, vol. 17, exp. 149, fs. 43 ; AGN, Mercedes, vol. 17, exp. 150, f. 43.

39 AGN, Mercedes, vol. 11, fs. 265v-266 ; AGN, Mercedes, vol. 12, fs. 71-71v ; AGN, Mercedes, vol. 15, fs. 290-290v ; AGN, Mercedes, vol. 19, exp. 474, fs. 225-225v.

40 En el territorio de Acámbaro, los miembros de la élite indígena lograron mantener un papel y un estatus muy importante dentro de las comunidades, además del reconocimiento de tierras heredadas y de la adquisición de nuevas. Varios consiguieron el derecho de andar en haca o de tener una espada, prerrogativas normalmente reservadas a los españoles. 
relativamente simultanea en el conjunto de las circunscripciones, a partir de la mitad de los años 1550 - principio de los años 1560. Este periodo corresponde al principio del registro sistemático de las concesiones por la administración española. Sin embargo, posteriormente, la colonización agropecuaria hispánica siguió dinámicas y flujos distintos en cada sector ¿Se trata de un efecto de la documentación o de una realidad histórica? Como lo subrayamos ya, el prisma del corpus histórico no puede omitirse. Documentos de donación de tierras desaparecieron o nunca fueron registrados, y eso no solamente para las primeras décadas de la colonización. Si bien a partir de los años 1560, el proceso de repartición de las mercedes realengas sigue un protocolo estricto, se reconoce que una parte de la documentación no ha sido conservada: algunos años de registro desaparecieron de manera parcial o total (Hunter, 2010: 3; Sluyter, 2002: 98). Basándose en los trabajos de Simpson (1952), se estima que sólo el $75 \%$ de las mercedes registradas en Nueva España han sido preservadas. En la región de Acámbaro-Maravatío, el corpus documental general es voluminoso, pero muy desigual de un sector a otro. La pérdida de información es difícil de cuantificar, pero con certeza afectó todas las cabeceras, por lo tanto no permite explicar, por sí solo, los distintos grados de colonización agropecuaria identificados en los territorios.

Por otro lado, las características geográficas propias de cada territorio y la diversidad de los paisajes tuvieron una influencia directa sobre la repartición de los terrenos. El territorio de Ucareo y una amplia parte del de Taimeo se centran en la imponente Sierra de Ucareo, cuyas cumbres pasan los 2,900 m de altura (figura 2). Este sector se caracteriza principalmente por un fuerte buzamiento ${ }^{41}$, que alcanza $43^{\circ}$, lo que favorece la erosión de los suelos, que es acentuada por las fuertes lluvias estivales. Desde luego, la delgadez de los suelos y su naturaleza andesita y basáltica son poco favorables al desarrollo de la agricultura. Por supuesto, estas características no impiden esta actividad, ya que los tarascos creaban terrazas en las faldas de los cerros, pero las propiedades

41 Es importante subrayar que también se encuentran valles y planicies (muchas veces estrechas) en la Sierra de Ucareo. Una de ellas se destaca, el amplio valle de Jeráhuaro cubierto de sedimentos aluviales. 


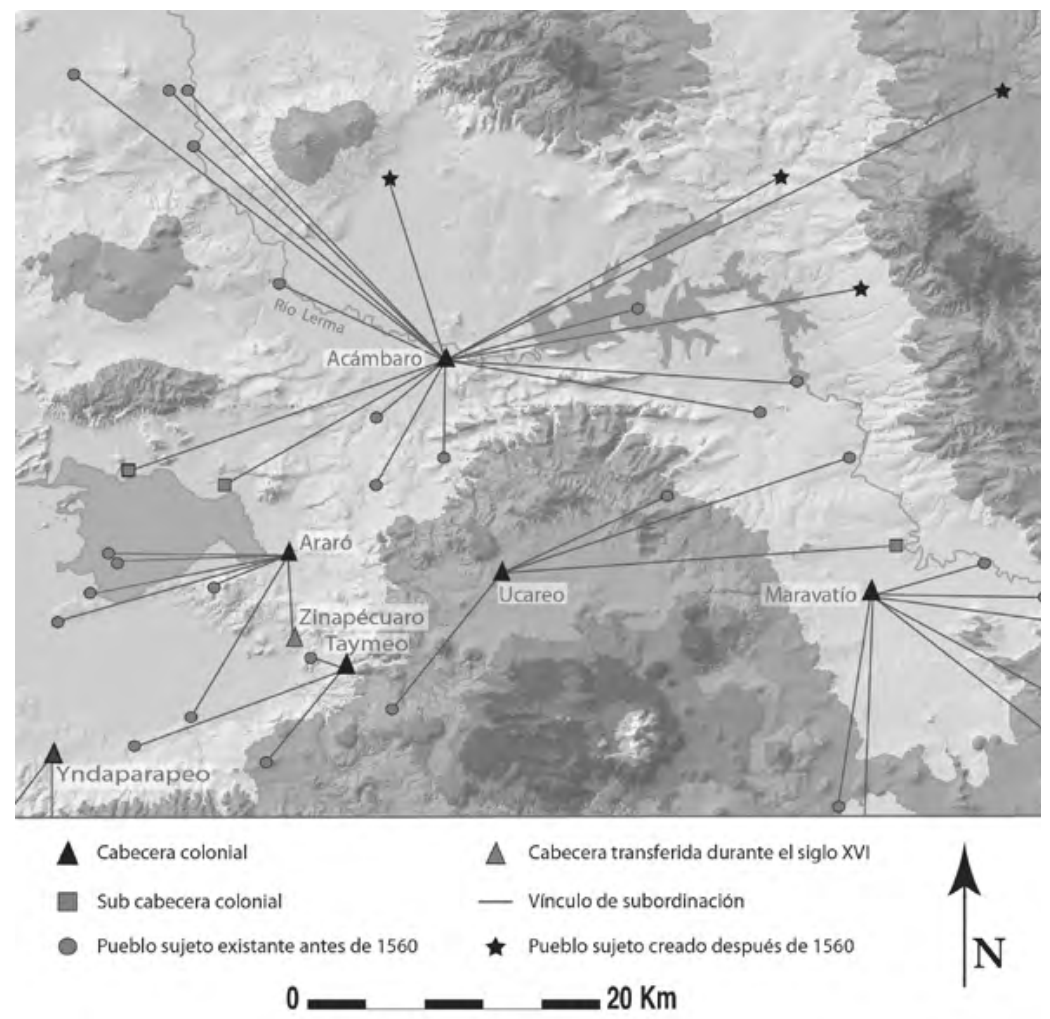

Figura $2 b$.

geológicas, en particular la calidad de los sedimentos, y la práctica exclusiva de la agricultura de temporal limitaban la productividad en comparación con los valles aluviales. Además, las terrazas, que requieren un mantenimiento regular, probablemente fueron afectadas por la caída demográfica. Estos factores podrían explicar que estos sectores interesaban menos a los españoles para el desarrollo de la actividad agrícola. En cambio, el escalonamiento favoreció el desarrollo de importantes bosques. A partir de los 2,000 m de altitud, las pendientes se cubren de robles y de pinos, lo que queda figurado en el documento cartográfico anteriormente mencionado (figura 4). Esta evocación no es cualquiera, ya que pone de manifiesto un recurso económico de primera 


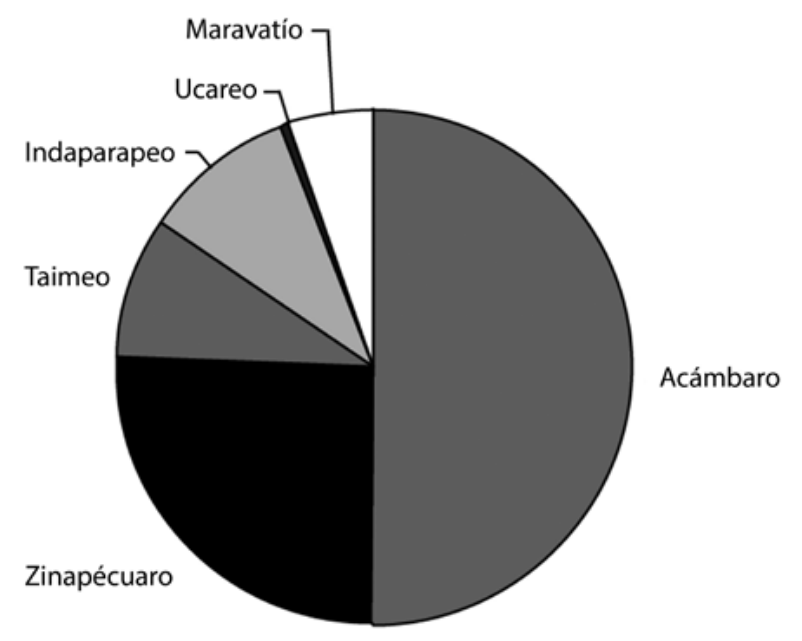

Figura 3. Repartición de las tierras otorgadas en cada cabecera (en número de mercedes).

importancia, sobre todo porque las especies leñosas de grandes dimensiones y por lo tanto, explotables para la carpintería, son repartidas de manera muy heterogénea en la región, puesto que los sectores bajos, llanuras y pequeños relieves, están cubiertos de una vegetación baja de tipo arbustiva. Numerosos documentos escritos y producidos en las primeras décadas del siglo XVI, subrayan la importancia de la explotación forestal realizada por las comunidades en la Sierra de Ucareo ${ }^{42}$. En cambio, no se registró explotación maderable a manos de españoles en esta época. ¿Este recurso sería exclusivamente en mano de las comunidades? Es difícil de saberlo. Lo que es seguro es que la geografía y la cobertura vegetal de la Sierra orientaron el patrón de asentamiento y el económico hacia la industria de la madera. El contexto económico, previo a la Conquista española, podría explicar el avance débil de la actividad agropecuaria hispánica en la zona.

42 AGN, Congregaciones, vol. único, exp. 164 fs 87v-88, AGN, Indios, vol. 6, exp. 92, fs. 23-23v. 


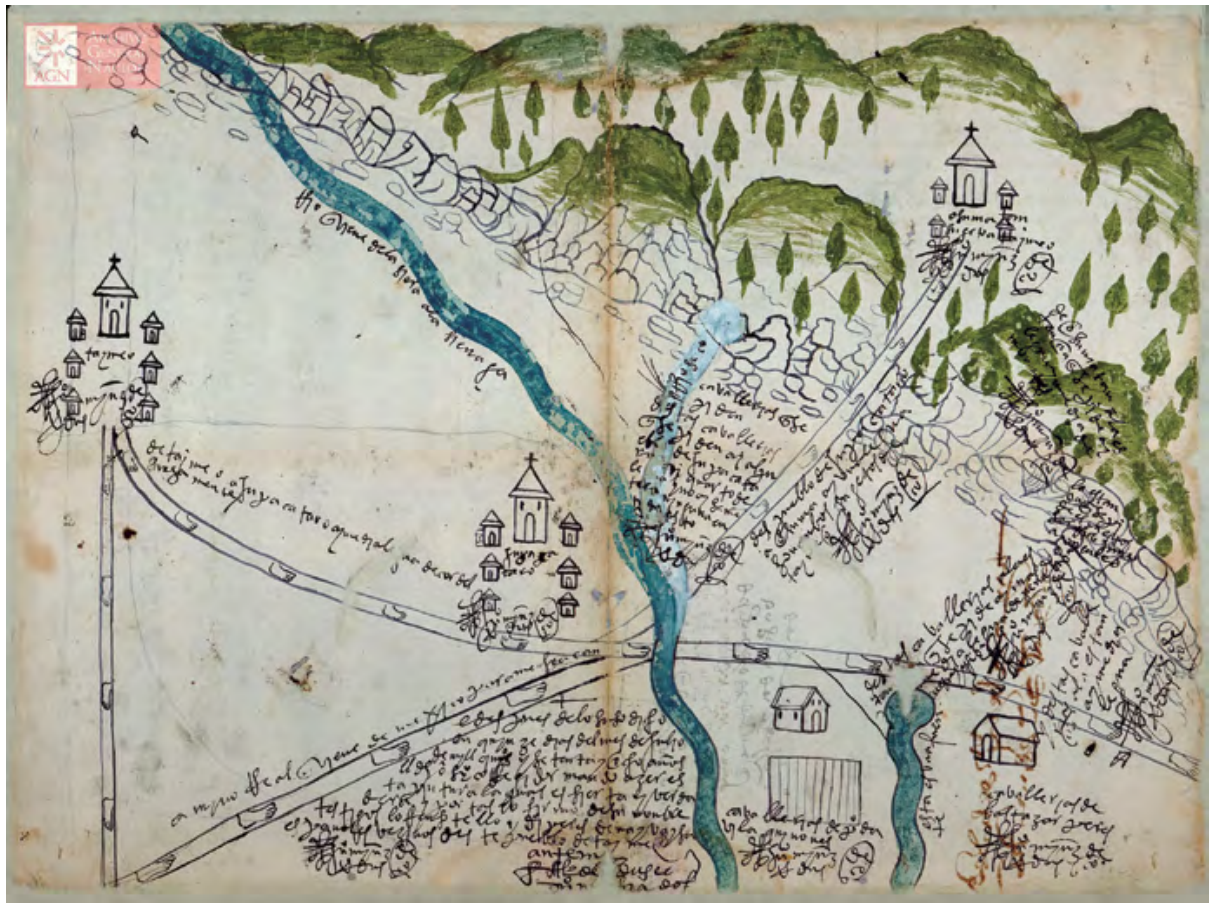

Figura 4. Fragmento de un territorio de Taimeo en 1578 (AGN, Tierras, vol. 3343, exp.I5, sección mapas, 2379).

Este sector montañoso contrasta con el valle del río Lerma y la Cuenca de Cuitzeo, en donde la ocupación hispánica está mucho más marcada. La topografía menos accidentada, la presencia de numerosos ríos y ojos de agua, y la gran fertilidad de los suelos formados por aluviones, son elementos particularmente favorables al desarrollo de una actividad agrícola ${ }^{43}$. Pero entonces, ¿`cómo explicar que la distribución de mercedes no haya sido más importante en los sectores de Maravatío y de Indaparapeo?, y ¿por qué los territorios de Zinapécuaro-Araró y Acámbaro fueron mucho más solicitados?

Además de la geografía, otros factores, esta vez antrópicos, tuvieron influencia en la colonización hispánica, tales como la demografía y la existencia

43 Mapas geológicos del INEGI: F14C84, F14C85, E14A14, E14A15. 


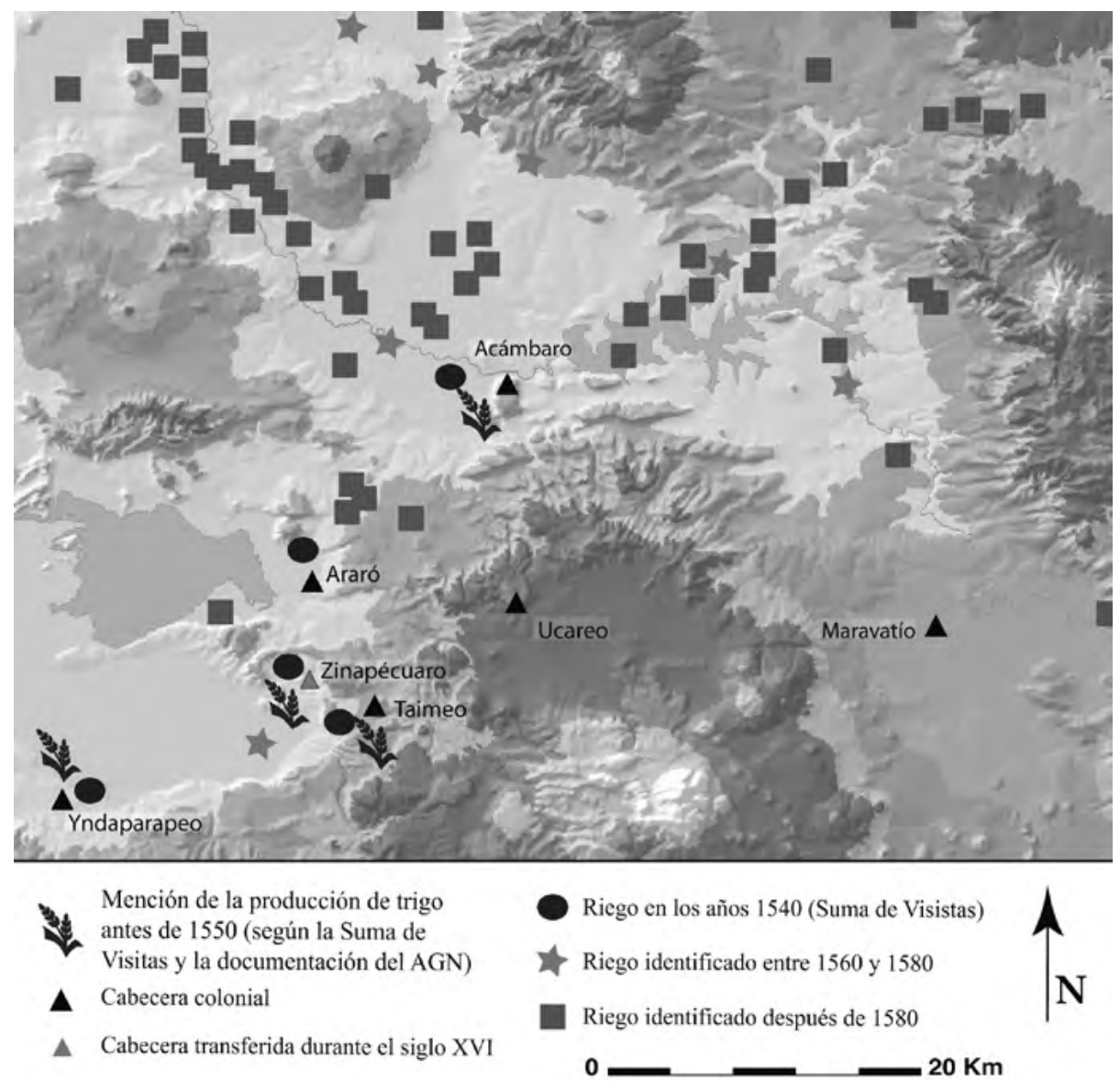

Figura 5. Mapa de las redes de riego del siglo XVI que pudieron ser ubicadas a partir de la documentación histórica.

de acondicionamientos agrícolas previos a la Conquista. Los cuatro territorios mencionados presentan una demografía desigual. La Suma de Visitas de los Pueblos (Paso y Troncoso, 1905) indica que, en 1548, el territorio de Maravatío contaba 579 habitantes, Indaparapeo: 637, Zinapécuaro-Araró: 829, y Acámbaro: 3,072, respectivamente $6,58 \%, 7,24 \%, 9,43 \%$ y $34,95 \%$ de la población total de la región. La baja demografía de Maravatío, Indaparapeo e 
Zinapécuaro-Araró podría ofrecer una magnífica oportunidad para los colonos hispánicos de ocupar tierras fértiles y "poco" explotadas (o en parte abandonadas). Pero, contrariamente, parece existir una correlación entre la demografía indígena y la fuerza de la implantación hispánica, siendo más importante esta última en los sectores de mayor densidad humana. Desde luego, la mano de obra explotable por los terratenientes se convierte en un factor determinante para escoger la zona de establecimiento de las parcelas. De hecho, la Suma de Visitas de los Pueblos evoca la requisición de las poblaciones locales para el trabajo agrícola. Por ejemplo, evocando el pueblo de Indaparapeo, indica que "Este pueblo tiene tres barrios y son todas las cassas noventa y seis y en ella ay seisçientas y treinta y siete personas de tres años para arriba; dan de tributo veynte y seis yndios de seruicio para las sementeras y huertas, y mas dan tres yndios que guardan las ouejas en el pueblo [...]" (Paso y Troncoso, 1905: 133). Esta fuente también menciona otro factor crucial: la existencia de sistemas de riego, apenas transcurridos 25 años de la Conquista, en los territorios de Acámbaro, de Araró-Zinapécuaro, de Taimeo y de Indaparapeo, es decir en los sectores de mayor difusión española. Los sistemas de riego prehispánico muchas veces fueron subestimados, y se atribuían al importante desarrollo hidráulico que ocurrió con la ocupación española. Lo anterior se debe a la falta de interés por los sistemas de riego y a la dificultad de evidenciarlos. La arqueología generalmente no proporciona informaciones satisfactorias sobre este tema, ya que las investigaciones se enfocan sobre todo en las zonas residenciales o rituales. Por cierto, los sectores agrícolas, y en particular los canales de riego, son difíciles de poner de manifestó, y necesitan la apertura de amplias superficies fuera de las zonas residenciales. Por otro lado, cuando son localizados, son difíciles de fechar. Estos acondicionamientos son explotados durante amplios periodos, y necesitan un mantenimiento y una limpieza constante para que no se obstruyan, lo cual afecta su estructura (ancho y profundidad) y enrarece el material arqueológico (ya escaso) que podría permitir fecharlos. En el mejor de los casos se puede dar un fechamiento postquem y antequem, en función de las unidades estratigráficas que los canales “cortan” y de las que los “tapan”. Las fuentes históricas también proporcionan informaciones limitadas. Como lo menciona B. Boehm Schöendube (2002: 48), las crónicas de los religiosos y de los 
visitantes, las tasaciones tributarias y las Relaciones Geográficas son generalmente imprecisas, evocando la presencia de riego a escala regional o microregional. Los documentos de los ramos Mercedes y Tierras del AGN permiten un nivel de estudio más local, haciendo referencia de manera más o menos directa al riego: "el remanece del agua del rio que viene del pueblo de Santiago Tarandacuao para con ella regar las tierras que tienen de sus comunidad" 44 , "dos cavallerias de tierra de riego" 45 , "quatro cavallerias de tierras con el agua del rio de Acámbaro para el riego dellas" 46 o simplemente por su ubicación "las caballerias junto a el rio grande" ${ }^{47}$. El tipo de vegetación cultivada también puede dar una indicación, ya que algunas especies no pueden crecer sin un aporte de agua importante. Estas menciones permiten la elaboración de un mapa parcial de la repartición de los sistemas de riego y de su evolución en el tiempo (figura 5). Pero, cabe subrayar que en la mayoría de los casos, estos documentos no especifican si el riego existía en la época prehispánica o si resultó de la llegada de los españoles. En el sector de Acámbaro, un documento con fecha de 1542 evoca un acuerdo sellado entre Hernán Pérez de Bocanegra y los indios de Acámbaro para "[...] que las tierras del dicho Pérez de Bocanegra tiene labradas, se las deja a los dichos indios de Acámbaro y los de Acámbaro asi mesmo dejan al Sr. Hernán Pérez las acequias que tienen sacadas y tierras labradas, para que ninguna de las partes desde éste día en adelante, se entre en las de la otra $[\ldots]^{\prime 48}$. Este evento sucede menos de 15 años después de la llegada de los españoles a la región, y con certeza hace referencia a sistemas de riego ya existentes previamente a la Conquista.

A diferencia de los sectores vecinos, no se encontró ninguna mención de la existencia de canales de riego prehispánicos para el territorio de Maravatío. De hecho, es posible que este sector, poco poblado y que no fue lo suficientemente explotado bajo la dominación tarasca, haya seguido un destino similar durante las primeras décadas de la colonia.

44 AGN, Mercedes, vol. 30, fs. 118v.

45 AGN, Mercedes, vol. 12, fs. $27 \mathrm{v}-28 \mathrm{v}$.

46 AGN, Mercedes, vol. 12, fs. 150v-151.

47 AGN, Mercedes vol 17, exp. 538, f. 150.

48 BN de Madrid, Sección Manuscritos, Manuscrito 11676, f. 9 in Escobar (1984 : 206). 
Así, con certeza, no sólo las características geográficas, sino también la importancia numérica de la población indígena y el grado de explotación agrícola en vísperas de la Conquista (y con eso los acondicionamientos existentes) influyeron sobre la colonización agropecuaria hispánica y su intensidad.

\section{LA COLONIZACIÓN AGROPECUARIA DEL TERRITORIO DE ACÁMBARO}

La colonización agropecuaria hispánica en el territorio de Acámbaro supone un caso singular en el panorama, tanto por su importancia numérica, como por la forma en que se dio. Durante la época colonial, el territorio administrativo de Acámbaro era mucho más amplio que él de las otras cabeceras, lo que seguramente explica, en parte, la desigualdad cuantitativa en la distribución de las tierras (figura 2). A la llegada de los conquistadores, el territorio de Acámbaro era ya muy extenso, pero contenido en la orilla sur del Lerma, así cubría una parte de los municipios actuales de Acámbaro, Salvatierra, Tarandacuao. Si bien, por lo general, la Corona solía apoyarse en la organización preexistente para asentar su dominio sobre las poblaciones locales, en algunas ocasiones se introdujeron cambios en la estructura administrativa o en los límites de los territorios. Es lo que sucedió con el territorio de Acámbaro, con el fin de facilitar el avance de los españoles y la colonización de nuevas regiones. Menos de 50 años después de la Conquista, se anexó a esta circunscripción una amplia extensión de tierra ubicada más allá del río Lerma, correspondiente a los municipios actuales de Jerécuaro, Coroneo y Tarimoro. Esta transformación de la organización administrativa es una consecuencia directa de la situación prehispánica. En víspera de la Conquista, esta región jugaba un papel de frontera del reino tarasco frente al imperio mexica al este y a los territorios chichimecas al norte, marcando así el límite del área mesoamericana. En la orilla sur del río, se establecieron grupos sedentarios: los tarascos y con ellos, grupos otomíes, mazahuas, matlatzincas que se reconocían vasallos del Cazonci (soberano tarasco). En cambio, el otro lado del río Lerma (norte) quedó vacío de ocupación, sirviendo de zona tapón entre los distintos grupos (Lefebvre, 2011). Esta situación explica que las seis cabeceras coloniales de la región, las cuales resultan de la organización administrativa prehispánica, se encuentran en la orilla sur 
del río Lerma. Apenas medio siglo después del establecimiento de los españoles, la "tierra de nadie" (orilla norte) fue unida a la circunscripción más cercana, la de Acámbaro, para facilitar su colonización y sobre todo el avance (y el tránsito) hacia los territorios septentrionales en donde se habían descubierto minas de metales preciosos desde mitad del siglo XVI. El territorio incorporado es considerable, puesto que desde entonces la superficie de la circunscripción de Acámbaro se volvió superior a las de las cinco otras cabeceras reunidas (Maravatío, Ucareo, Taimeo, Araró-Zinapécuaro e Indaparapeo). La superficie sobredimensionada del territorio explica en parte la abundancia de tierras otorgadas en Acámbaro y en sus pueblos sujetos. Pero, más allá del criterio numérico, es el proceso de colonización agropecuario que se vio afectado por esta situación peculiar. La diferencia de contexto histórico y social entre las orillas sur y norte, necesariamente tuvo una influencia en el desarrollo de la colonización, ya sea en sus aspectos cronológicos como en los mecanismos sociales y económicos. A principios del siglo XVI, el sector sur, ocupado por las poblaciones sedentarias, estaba marcado por un paisaje antropizado, caracterizado por la presencia de pueblos, de terrazas agrícolas en las faldas de los cerros, de parcelas agrícolas trabajadas o de presas, que podían explotar los colonos. Pero, los recién llegados debían de insertarse en una red de población y de terratenientes ya establecida. Por el contrario, la orilla norte se encontraba desertificada desde hacía por lo menos un siglo y medio, lo que provocó el abandono de sus terruños y la aniquilación progresiva de los acondicionamientos. Esta situación ofreció a los españoles un espacio "casi virgen” y por lo tanto una mayor libertad en cuanto al reparto y a la explotación de las tierras, pero en contraparte impuso un esfuerzo considerable para el establecimiento (o re establecimiento) de las obras hidráulicas y otras estructuras necesarias para el desarrollo de las actividades agropecuarias. El paisaje es un producto social resultante de siglos de ocupación humana, del marco mental de las sociedades que se sucedieron en él, de su organización social, sus coacciones productivas, de sus avances tecnológicos, entre numerosos factores. Así, dos zonas "geográficamente similares" que sufrieron procesos históricos distintos presentarán un paisaje cultural diferente. Por lo tanto, si viene a establecerse una misma población en ellos, cada uno de estos sectores, de estos 
paisajes, tendrá una incidencia propia sobre la sociedad, orientando su economía, su patrón de asentamiento... Por consiguiente, es interesante observar con más atención las características de la difusión de las poblaciones europeas en el territorio de Acámbaro.

En la orilla sur del río Lerma, la presencia de terratenientes españoles está atestada por lo menos desde 1542, cuando el encomendero Hernán Pérez de Bocanegra intercambia tierras con los naturales de Acámbaro. Pero, como en las demás cabeceras, hay que esperar a los años 1560 para que se registren (y se multipliquen) las mercedes. Si bien las primeras unidades productivas europeas fueron silenciadas, y por lo tanto no pueden ser ubicadas, tanto temporal como espacialmente, los litigios registrados ponen de manifiesto la proximidad de las tierras otorgadas con los asentamientos indígenas. No obstante, en esta época, los poblados se concentran en la orilla sur, en el sector ocupado bajo la dominación tarasca, lo que permite afirmar que unas parcelas también se encontraban en esta zona. Hay que esperar la década de 1560 para que se asienten los primeros poblados en la orilla norte. No existen actos de fundaciones para estos asentamientos nuevos, pero la primera mención de su existencia en las fuentes escritas da una indicación: Puruagua $(1563)^{49}$, Jerécuaro $(1565)^{50}$, Coroneo $(1580)^{51}$, Paraquaro $(1580)^{52}$. En 1560, la merced de un sitio de estancia fue otorgada en la orilla norte, a proximidad del camino real que va a Querétaro $^{53}$, marcando el principio de la colonización agropecuaria de este sector. Por la falta de registro sistemático de las tierras agrícolas anterior a 1560, es imposible afirmar que se trata de la primera merced en esta orilla norte. Pero, la fecha es coherente con la aparición de los asentamientos en este sector. Desde luego, empieza la repartición de la antigua zona tapón. Dentro de las 71 parcelas (de un total de 95) que pudieron ser localizadas con una cierta precisión gracias a los elementos geográficos y terrenos circunvecinos mencionados para

49 AGN, Mercedes, vol. 7, fs. 120-120v. Este pueblo, como los siguientes, no aparecen en las listas tempranas de pueblos sujetos.

50 AGN, Mercedes, vol. 8, fs. 53-53v.

51 Acuña (1987, p. 62).

52 Acuña $(1987$, p. 62).

53 AGN, Mercedes, vol. 5 , fs. $11 \mathrm{v}$. 
delimitar las parcelas, 31 se encuentran en la orilla norte y 40 en la sur ${ }^{54}$. Las parcelas de la orilla norte se entregaron casi exclusivamente a colonos españoles, pero algunos indios de alto estatus, caciques o principales, también lograron salir adelante en este sector. En 1584, don Diego del Águila, poderoso cacique de Acámbaro, obtiene tierras al pie del cerro Urireo, cerca de Salvatierra $^{55} .10$ años después, enriquece sus posesiones con dos tierras ganaderas en la llanura del Jaral, próximas a las propiedades ya en sus manos ${ }^{56}$. En 1590, don Zacarías igualmente recibe un sitio de estancia para la cría de ganado menor y dos caballerías de tierra en la llanura del Jaral, próximas a las tierras de don Diego del Águila. Si bien estas tierras concedidas a miembros de la nobleza indígena no son mayoritarias, ni siquiera numerosas, demuestran una particularidad de la repartición agraria. Sólo los españoles y los indios de alto nivel social obtienen tierras en la orilla norte del río Lerma. Las comunidades indígenas sólo recibieron parcelas agrícolas y ganaderas en la orilla sur (o en la ribera norte en las inmediaciones del río Lerma). En este caso, probablemente se trata del reconocimiento de antiguas posesiones más que de la repartición de nuevas propiedades. Para los caciques, el alejamiento de las parcelas puede ser más importante, aunque generalmente queda inferior a ocho $\mathrm{km}$ del río. Más allá, las tierras rodeando las aglomeraciones nuevas de Jerécuaro, Coroneo y Puruagua fueron "reservadas", o por lo menos otorgadas, a colonos europeos. Por lo tanto, este sector desocupado durante la época tarasca, se encuentra dominado por las poblaciones hispánicas a partir de la segunda mitad del siglo XVI.

Este esquema general de difusión de las poblaciones hispánicas dentro del amplio territorio de Acámbaro, así como la aparición tardía y escasa de los pueblos nuevos en la orilla norte, a lo largo de las vías de comunicación, deja vislumbrar una colonización planificada por las poblaciones hispánicas. En

54 Nueve parcelas no pudieron ser ubicadas siguiendo el reparto orilla sur/orilla norte, porque las indicaciones geográficas solamente las ubican de manera muy general a nivel de las riberas del río Lerma. Las últimas 15 mercedes no presentan indicaciones suficientes para poder ubicarlas.

55 AGN, Mercedes, vol. 12, fs. 71-71v.

56 AGN, Mercedes, vol. 19, exp. 474, fs. 225-225v. 
efecto, en un primer tiempo, durante las cuatro primeras décadas de presencia hispánica, se identifica una ocupación exclusiva en la orilla sur, a través de una explotación de las tierras abandonadas por las poblaciones indígenas por culpa de la epidemias o de las políticas de congregaciones (o directamente usurpadas a las comunidades). Sólo en un segundo tiempo, se extiende la colonización agropecuaria en los territorios septentrionales.

La distribución funcional de las parcelas también aporta elementos para entender la colonización agropecuaria. Si consideramos el número de propiedades otorgadas (y no la superficie ${ }^{57}$ ), $65,26 \%$ de las mercedes concedidas en el conjunto del territorio son destinadas a la explotación agrícola (unos 13,800 ha.). Las caballerías de tierras representan $55,67 \%$ de las tierras otorgadas en el territorio de Zinapécuaro, 52,72 \% en Taimeo, 66,6 \% en Indaparapeo y 71,47 \% en Acámbaro. Esta vocación privilegiada se explica por la gran fertilidad de los suelos y las numerosas fuentes de agua en los sectores "bajos" donde fueron otorgados la gran mayoría de las tierras, pero sobre todo, por la gran necesidad de sus producciones agrícolas. En efecto, si bien una amplia parte de las producciones agrícolas era destinada al consumo de las poblaciones establecidas en los centros urbanos nacientes y en los centros mineros, una porción, que no es despreciable, sirvió de complemento alimenticio al ganado, retroalimentando el sistema agropecuario. El análisis del corpus de mercedes puso de manifiesto que con frecuencia, españoles, caciques y comunidades obtenían conjuntamente una estancia para la cría del ganado (menor o mayor) y dos caballerías de tierras. Algunos documentos denuncian de manera directa la vocación de estas parcelas agrícolas. En 1563, Hernando Pantoja obtiene “[...] una caballería de tierra en termino de Yndaparapeo para el sustento de una estancia de ganado menor que tiene [...]" ${ }^{58}$. El mismo año, Lope Cendejas recibe "[...] un sitio de estancia para ganado mayor con caballerías y media de tierra para el sustento della [...]" 59 . A pesar del tamaño importante de las tierras

57 Recordamos que las parcelas otorgadas tienen una superficie distinta según la función que se le concedía. Por consiguiente, es esencial distinguir el número de tierras otorgadas de su superficie efectiva.

58 AGN, Mercedes, vol. 7, f. 128.

59 AGN, Mercedes, vol. 7, f. 264. 
ganaderas (780 ha para las estancias de ganado menor y 1,755 ha para las de ganado mayor), parece que el aporte de un complemento alimenticio para las manadas era indispensable o por lo menos bastante común para que esta asociación fuera facilitada por las autoridades españolas.

De nuevo, el caso muy específico de Acámbaro demuestra que la repartición de las caballerías y de las estancias varía en el espacio y en el tiempo. En la orilla sur, antiguo reino tarasco, el 74,4\% de las tierras otorgadas (que pudieron ser ubicadas) es agrícola, y esta proporción parece estable a lo largo del tiempo. En cambio, en el sector norte, la proporción de tierras agrícolas es más baja, y representa el $62 \%$ de las tierras otorgadas. Pero, la principal distinción viene de la repartición temporal de las mercedes. Hasta los años 1565, el conjunto de las tierras otorgadas están dedicadas a la ganadería. Sólo a continuación, se van a introducir las primeras tierras agrícolas y se van a equilibrar las proporciones para alcanzar la tendencia general con un sitio de estancia por dos sitios de caballería. El establecimiento de estas nuevas parcelas agrícolas siempre se caracteriza por la cercanía de ríos: “[...] dos caballerías de tierra dentro del termino della en terminos del pu[ebl]o de Acámbaro y de Chupícuaro junto a el arroyo que viene del pu[ebl]o de Xerequaro [...]"60, “[...] de dos caballerías de tierra en términos del pu[ebl]o de Acámbaro en el rio de San Miguel [...]"61 " "[...] y de caballería y media de tierra en el rio de Apaçeo [...]"62. Esta evolución resalta la introducción progresiva de obras hidráulicas y al mismo tiempo de la actividad agrícola.

Cabe notar que el territorio de Maravatío sigue un destino similar. El conjunto de las tierras otorgadas antes de 1560 , tenían una función ganadera ${ }^{63}$. La primera parcela agrícola se atribuye en $1561^{64}$. Este elemento conforta las conclusiones evocadas previamente, en cuanto a la ausencia o más bien a la baja inversión agrícola notable en este sector previamente a la Conquista. Ahí

60 AGN, Mercedes, vol. 8, fs. 53-53v.

61 AGN, Mercedes, vol. 8, fs. 118-118v.

62 AGN, Mercedes, vol. 7, fs. 120-120v.

63 AGN, Mercedes, vol.4, exp. 198, f. 62 ; AGN, Mercedes, vol. 4, exp. 218, f. 70v; AGN, Mercedes, vol. 4, f. 261.

64 AGN, Mercedes, vol. 5, fs. 217v. 
también, en un primer tiempo la ganadería está más desarrollada, y sólo en un segundo tiempo se desarrollan las estructuras de riego, o por lo menos llegan a manos de los colonos.

\section{ENTRE CAMPOS Y CORRALES}

La propagación de la colonización agropecuaria es calculada. El objetivo es el aprovechamiento de las infraestructuras indígenas preexistentes (antes de la caída del reino tarasco). Por lo tanto, no es anodina, está destinada a favorecer el desarrollo de las unidades productivas en manos de europeos, permitiéndoles ser productivos casi inmediatamente. En efecto, el cultivo del trigo está atestiguado de manera muy temprana en la región. Como lo mencionamos ya, el primer acto de merced inventariado por el sector con fecha de 1542, concede a Hernán Pérez de Bocanegra un sitio por la edificación de un molino y un área de batán en el territorio de Zinapécuaro ${ }^{65}$. Si bien la palabra "trigo" no esta mencionada, se trata de la primera referencia al cereal europeo en la región, puesto que es el único producto que necesita este tipo de molienda. Es imposible decir si el trigo procede de campos en manos de la familia Bocanegra o de las comunidades indígenas, como una producción impuesta a las poblaciones. El encomendero podía adquirir esta producción por medio del tributo que pagaban las comunidades. Pero, también es posible que la producción viniera de los campos de riego, intercambiados con los naturales de Acámbaro el mismo año.

La Suma de Visitas proporciona otro testimonio, esta vez directo, de la integración temprana del cereal europeo en los campos. En este documento, fechado de 1548, el trigo aparece junto al maíz en las cargas tributarias concedidas por las poblaciones indígenas a los encomenderos de Acámbaro, de Indaparapeo y de Taimeo (Paso y Troncoso, 1905: 33, 133 et 252). Por consiguiente, desde antes de 1550, el trigo ya está presente en cuatro cabeceras y era impuesto a las comunidades por los colonos que no deseaban adaptarse al régimen alimenticio prehispánico. La repartición de las zonas de producción del trigo corresponde no solamente a las tierras más fértiles, pero también

65 AGN, Mercedes, vol.1, exp. 226, f. 105. 
coincide con la difusión de los sistemas de irrigación durante el Posclásico tardío según las fuentes escritas. Estos acondicionamientos agrícolas facilitaron la introducción del cereal europeo, que no era adaptado al ritmo estacional mexicano, ya que su crecimiento se desarrolla durante la temporada seca, es durante este periodo que su necesidad de agua es mayor. Bajo un clima tropical, tal como es el caso en la región de Acámbaro, la irrigación resulta ser un factor imprescindible para cultivar el trigo. Por consiguiente, los españoles explotaron los canales indígenas para favorecer la integración de vegetales importados desde el "viejo" mundo.

La colonización hispánica también introduce una nueva variable: la ganadería, ausente durante la época prehispánica, y que va a hacerse una plaza transformando profundamente el paisaje. Su introducción no fue sin conflictos. Estos litigios fueron numerosos en la región de Acámbaro y oponían principalmente las comunidades indígenas y los colonos españoles. Los documentos históricos atestiguan de los perjuicios imputables a las manadas, y principalmente al ganado mayor que pisaba las sementeras de los indios, destruyendo sus cosechas. La primera mención de ganado en la región corresponde a la compra de estancias por el virrey Antonio de Mendoza en el territorio de Maravatío. A partir del año siguiente, también aparecen los primeros litigios, esta vez en el territorio del Zinapécuaro: el primer acto identificado, fecha de 1542, y menciona la presencia de animales en los campos de la comunidad ${ }^{66}$. Este documento testimonia la difusión muy temprana del bestiario europeo. Esta situación conflictiva entre agricultores y ganaderos se persigue durante varias décadas. Todavía en 1561, los indios de Acámbaro ${ }^{67}$, de Zinapécuaro ${ }^{68}$ y de Ucareo $^{69}$ sufrían una situación similar. En vísperas del siglo XVII, en 1591, los habitantes de San Bartolomé (un pueblo sujeto de Zinapécuaro) se quejaron de que "[...] muchos Españoles traen mucha cantidad de ganados en sus términos y entre sus sementeras los quales les comen y destruyen y hazen muchos

66 AGN, Mercedes, vol.1, exp. 399, f. 186.

67 AGN, Mercedes, vol. 4, f. 282.

68 AGN, Mercedes, vol. 5, f. 293v.

69 AGN, Mercedes, vol. 5, f. 340. 
daños de manera que por no poder coger el fruto dellas padecen ambre y nessesidad [...] $\gg^{70}$. Sin embargo, cabe notar que a partir de los años 1560, los conflictos se vuelven menos frecuentes. Las razones de este apaciguamiento de las relaciones entre agricultores y ganaderos no están claramente mencionadas en las fuentes, pero muy probablemente se debe a la separación de las áreas de actividades, impuesta por las autoridades novohispanas. En un mapa realizado en $1616^{71}$ figuran las transformaciones que ocurrieron en el patrón agropecuario acámbarense, casi un siglo después de la Conquista (figura 6). Esta pintura presenta claramente la repartición de las tierras en áreas específicas: la presencia de campos, probablemente de riego, a lo largo del río Lerma, de campos (de temporal) rodeando los pueblos "nuevos" de indios, y la segregación de las estancias (estilizadas por los palos que conforman el cercado), relegadas en los cerros. En la documentación escrita, las descripciones de los límites de las parcelas refuerzan esta distribución espacial. Por ejemplo, en 1575, un español, Diego Pérez obtiene una estancia localizada "[...] en la sierra que dizen de Barajas [...]" y dos caballerías de tierra a proximidad del río de San Miguel ${ }^{72}$. De la misma manera, en 1583, un tal Francisco Rodríguez recibe un sitio para la cría de ganado mayor y dos tierras agrícolas. El acto de donación especifica que el pastizal se encuentra "[...] a la [f]alda de un cerrillo pedregoso y a la [f] alda de un cerro grande donde esta un charco de agua [...]"73. Así, se establecieron políticas de colonización agropecuaria, aspirando a reducir el número de litigios y a estructurar el paisaje colonial, en vista de un mejor aprovechamiento. Desde luego, aparecen sectores específicos dedicados a una u otra actividad en función de sus características geográficas y geológicas. De manera privilegiada, las tierras agrícolas se encuentran en las llanuras o en los valles para facilitar el trabajo de la tierra, y en la medida de lo posible, próximas a un ojo de agua o un río para favorecer el establecimiento de sistema de riego, mientras que las parcelas ganaderas fueron relegadas en las laderas de los

70 AGN, Indios, vol. 5, exp. 962, fs. 317v-318.

71 AGN, Tierras, vol. 2735, $2^{\circ}$ parte, exp. 2, f.10, sección mapas, 1928.

72 AGN, Mercedes, vol. 8, fs. 110v-111.

73 AGN, Mercedes, vol. 12, fs. 27v-28. 


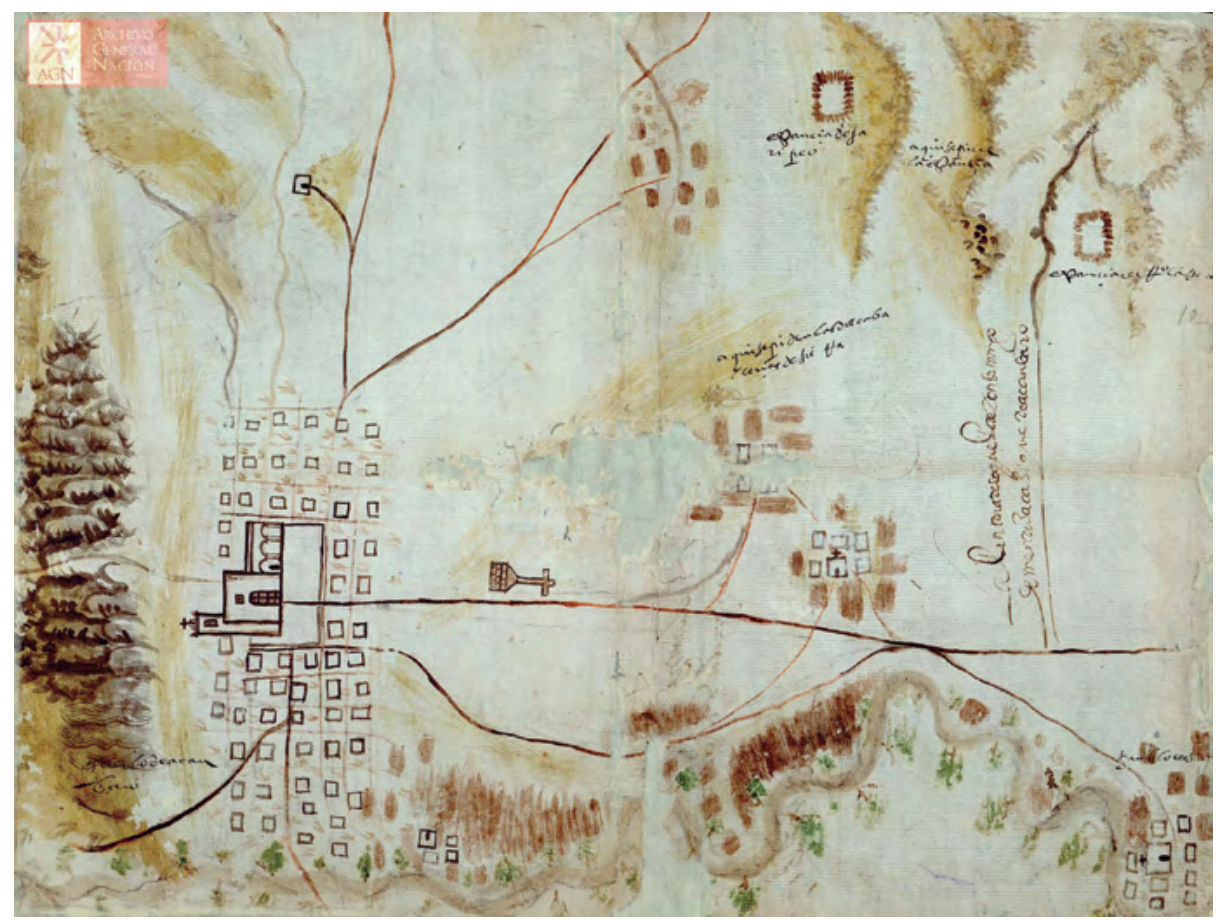

Figura 6. Distribución de las tierras productivas en el territorio de Acámbaro en I6ı6 (AGN, Tierras vol. 2735, $2^{\circ}$ parte, exp. 2, f.Io, sección mapas, I928)

pequeños cerros y de las sierras. Una situación similar fue demostrada en la región de Chalco por la investigación de T. Jalpa Flores (2008). Se trata de una cambio mayor ya que bajo la dominación tarasca, una parte de la producción agrícola se produce en las faldas, entonces cubiertas de terrazas agrícolas, rodeando los antiguos asentamientos, tal como se puede observar en el sitio de Cerro el Toro (Acámbaro), Tocuaro, El Pedrillo (Zinapécuaro), por mencionar solo algunos. Por lo tanto, la transformación del patrón agropecuario siguió los pasos de los desplazamientos de los pueblos indígenas.

Desde luego, esta distribución espacial de las actividades agropecuarias tiene un doble interés. En primer lugar, limita las invasiones animales en los campos y por lo mismo la destrucción de las cosechas. Por otra parte, permite una mejor rentabilización del territorio, utilizando las tierras más fértiles y 
adecuadas para la agricultura y optimizando las zonas menos productivas. Sin embargo, algunos sitios ganaderos parecen escapar a esta regla, y se establecen en llanuras próximas a ríos, que servían entonces como abrevadero para las manadas, o en zonas pantanosas, en donde los animales encontraban una abundante vegetación para alimentarse. Por ejemplo, en 1582, Joachim de Losu percibe un sitio de ganado menor y dos caballerías de tierras cerca de Zinapécuaro, entre la laguna y el camino real que lleva a Valladolid ${ }^{74}$. La implantación de estancias en el sector pantanoso demuestra que la naturaleza de los suelos, favorable a la agricultura, no es un freno a la explotación ganadera. Joachim de Losu fue teniente de alcalde mayor de Zinapécuaro, su estatus privilegiado podría explicar su adquisición.

\section{CONCLUSIÓN}

El análisis de la documentación administrativa demuestra que el proceso de difusión de las poblaciones hispánicas en las "nuevas provincias" y la intensidad de la colonización agraria varió en el tiempo y en el espacio. Se identificaron cuatro fases de repartición de las tierras, entre las cuales se destacan dos momentos de auge, el primero entre 1560 y 1568 y el segundo de 1581 a 1596 . En un primer tiempo, hasta finales de los años 1570, la invasión española es limitada, y se restringe principalmente a los conquistadores, a los miembros de la administración local o de la alta administración y a su familia respectiva. Estos individuos rápidamente se convierten en "poderosos", favorecidos en la adquisición de propiedades, recibiendo tierras en gran número o en lugares particularmente propicios por la fertilidad de las tierras, la presencia de agua e incluso, la cercanía con caminos importantes que permiten las salidas económicas. Por otro lado, su alto estatus también les proporcionó una posición, que aprovecharon para usurpar tierras a las comunidades. El segundo pico de repartición, cuya magnitud es sin paragón con el primero, se caracteriza por la llegada "masiva" de una nueva clase de terrateniente sin vínculo ninguno con la Conquista. Estos particulares, en búsqueda de riqueza y de un mejor estatus,

74 AGN, Mercedes, vol. 11, fs. 156-156v. 
vienen a sumirse a las "viejas" familias de conquistadores que siguen obteniendo propiedades.

Los flujos de difusión de las poblaciones europeas en la provincia están vinculados con las caídas demográficas de las poblaciones indígenas (por culpa de las epidemias, del trabajo forzado y de los conflictos con los españoles) y las transformaciones del patrón de asentamiento. En efecto, los momentos de mayor distribución de las tierras coinciden con la primera fase de congregación y los picos epidémicos que "liberaron" tierras, las cuales fueron rápidamente acaparadas por los colonos.

A una escala más local, se identifica que las seis cabeceras que integran la región de estudio presentan destinos distintos frente a esta invasión. Los colonos se difundieron de manera calculada, aprovechando los beneficios naturales que podían ofrecer cada sector. Así, los valles y llanuras, en los cuales se encuentran sedimentos aluviales fértiles y numerosos cuerpos de agua y manantiales, fueron particularmente repartidos. Pero también, se tomaron en cuenta las características antrópicas preexistentes a la llegada de los españoles, tales como la importancia demográfica, que permitía proporcionar una mano de obra suficiente para el trabajo de las parcelas o para cuidar el ganado, y la existencia previa de acondicionamientos agrícolas, en particular de redes de irrigación que se volvieron un elemento decisivo ya que favorecieron la introducción temprana de especies europeas, como el trigo. Por lo tanto, se observa un primer establecimiento en los sectores no solamente ocupados durante el Posclásico tardío, sino también que ya disponían de un sistema agrícola elaborado. En lo demás del territorio, que carecía de estos equipamientos (territorio de Maravatío y sector septentrional), la actividad ganadera fue privilegiada en un primer tiempo, puesto que no requería acondicionamientos específicos y se podían aprovechar amplias extensiones de tierra como pastizales. Solo en un segundo tiempo se introduce, o se desarrolla, la agricultura extensiva. Eso demuestra una colonización agropecuaria pensada y planificada para aprovechar cada paisaje en función de sus características naturales y culturales.

La colonización española tuvo repercusiones profundas y prontas en el área. Los cambios de tenencia de la tierra y de uso de suelo, las alteraciones sociales y las transformaciones de la económica agrícola al origen de la creación 
de nuevos acondicionamientos (tales como la ampliación de las redes de riego, la reestructuración del parcelar) y del abandono progresivo de otros (como las terrazas agrícolas), pero también la desecación de cuerpos de agua y la tala de bosques con el fin de ampliar las tierras agropecuarias, son el origen de la emergencia de un paisaje colonial, o mejor dicho, de paisajes coloniales. A la manera de un palimpsesto (para utilizar la expresión establecida), los paisajes, entendidos como espacios dinámicos de vestigios culturales sobrepuestos, sufrieron fenómenos complejos de transmisión y de recomposición, guardando la memoria de su pasado prehispánico, al mismo tiempo que se inscribían la huella de los nuevos "poderosos".

\section{BIBLIOGRAFÍA}

- Acuña, R. (1987). Relaciones Geográficas del siglo XVI: Michoacán. México: UNAM, Instituto de Investigaciones Antropológicas.

- Boehm Schoendube, B. (2002). El riego prehispánico en Michoacán. En M. Sánchez R. (coord.). Entre campos de esmeralda. La agricultura de riego en Michoacán (pp. 47-75). Zamora, Michoacán: El Colegio de Michoacán /Gobierno del Estado de Michoacán.

- Butzer, K. W. y E. K. Butzer (1993). The sixteenth-century environment of the central Mexican Bajío: Archival reconstruction from colonial landgrants and the question of Spanish ecological impact. Geoscience and Man 32, 80-124.

- Cook, S. F. y L. B. Simpson. (1948). The population of Central México in the sixteenth century. Berkeley, EUA: Ibero-Americana/University of California Press.

- Endfield, G.H. y S. L. O'Hara. (1999). Degradation, Drought, and Dissent: An Environmental History of Colonial Michoacán, West Central Mexico. Annals of the Association of American Geographers 89, 402-419.

- Escobar Olmedo, A. (1984). Las encomiendas en la cuenca lacustre de Cuitzeo. En C. Paredes Martínez, M. Piñón Flores, A. Escobar Olmedo y M. Pulido Solís (eds.), Michoacán en el siglo XVI. (pp. 191-295). Morelia: Fimax Publicistas.

- Fernández-Christlieb F. y P. Urquijo. (2006). Los espacios del pueblo de indios tras el proceso de congregación, 1550-1625. Investigaciones Geográficas. Boletín del Instituto de Geografía 60, 145-158. 
- Hunter, R. (2009). People, sheep, and landscape change in colonial Mexico: The sixteenth-century transformation of the Valle del Mezquital. Phd diss./Louisiana State University.

- Hunter, R. (2010). Methodologies for Reconstructing a Pastoral Landscap, land grants in sixteenth-century New Spain. Historical Methods A Journal of Quantitative and Interdisciplinary History 43(1), 1-13.

- Jalpa Flores, T. (2008). Tierras y sociedades: la apropiación del suelo en la región de Chalco durante los siglos XV-XVII. México: INAH.

- Lefebvre, K. (2011). Acámbaro, en los confines del reino tarasco: una aculturación discreta (1440- 1521 d. C.). Trace 59, 74-89.

- Lefebvre, K. (2012). L'occupation du sol dans la région d'Acámbaro entre le Post-

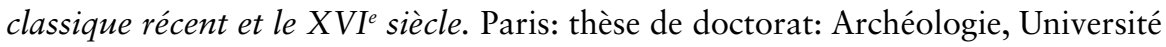
de Paris I, Panthéon - Sorbonne, 2 vol.

- Melville, E. (1994). A Plague of Sheep: Environmental Consequences of the Conquest of Mexico. Cambridge: Cambridge University Press.

- Musset, A. (2002). Villes nomades du nouveau monde. Paris: Éditions de l'École des Hautes Etudes en Sciences Sociales.

- Paso y Troncoso, F. (1905). Suma de visitas de pueblos por orden alfabético, manuscrito 2,800 de la Biblioteca Nacional de Madrid, anónimo de la mitad del siglo XVI. En Papeles de la Nueva España. Segunda serie, geografía y estadística. Madrid: Est. Tipográfico "Sucesores de Rivadeneyra", t. I.

- Reyes García, C. (2003). Las republicas de naturales del occidente de Michoacán. En C. Paredes Martínez y M. Terán, Autoridad y gobierno indígena en Michoacán. V. 1. (pp. 105-131). México: El Colegio de Michoacán/INAH/CIESAS/UMSNH,

- Roskamp, H. (2016). De la costumbre al abuso: el gobernador Alonso Huapean, Zinapécuaro, 1566-1567. En H. Roskamp y S. Albiez Wieck (Eds.). Nuevas contribuciones al estudio del antiguo reino tarasco. Zamora, Michoacán: El Colegio de Michoacán.

- Ruiz Medrano, E. (1991). Gobierno y sociedad en Nueva España: segunda audiencia y Antonio de Mendoza. Zamora, Michoacán: El Colegio de Michoacán, Gobierno del Estado de Michoacán. 
- Sánchez Rodríguez, M. y B. Boehm Schoendube. (2005). Cartografía Hidráulica de Michoacán. Zamora, Michoacán: El Colegio de Michoacán, Gobierno del Estado de Michoacán.

- Sánchez Rodríguez, M. y E. H. Jr Herbert. (2007). Cartografía Hidráulica de Guanajuato. Zamora, Michoacán: El Colegio de Michoacán/Consejo de Ciencia y Tecnología.

- Sauer, C. O. [1925] (2006). La morfología del paisaje. Polis. Revista Latinoamericana 5, [en linea]. [Fecha de consulta: 6 de noviembre de 2017] Disponible en: $<$ http://www.redalyc.org/articulo.oa?id=30517306019>

- Simpson, L. B. (1952). Exploitation of land in central Mexico in the sixteenth century. Ibero-Americana 36.

- Sluyter, A. (2002). Colonialism and Landscape: Postcolonial Theory and Applications. Lanham: Rowman and Littlefield.

- Torre Villar, E. de la. (1995). Las congregaciones de los pueblos de Indios en la Nueva España. México: UNAM, Instituto de Investigaciones Históricas.

- Worster, D. (1990). Transformations of the Earth: Toward an Agroecological Perspective in History. Journal of American History 76 (4), 1087-1106.

\section{LISTA DE FIGURAS}

- Figura 1: Evolución cronológica de la distribución de las mercedes (número de donación por año)

- Figura 2: Extensión de los territorios de las cabeceras durante el siglo XVI, según los datos históricos

- Figura 3: Repartición de las tierras otorgadas en cada cabecera (en número de mercedes)

- Figura 4: Fragmento de un territorio de Taimeo en 1578 (AGN, Tierras, vol. 3343, exp.15, sección mapas, 2379)

- Figura 5: Mapa de las redes de riego del siglo XVI que pudieron ser ubicadas a partir de la documentación histórica

- Figura 6: Distribución de las tierras productivas en el territorio de Acámbaro en 1616 (AGN, Tierras vol. 2735, $2^{\circ}$ parte, exp. 2, f.10, sección mapas, 1928) 